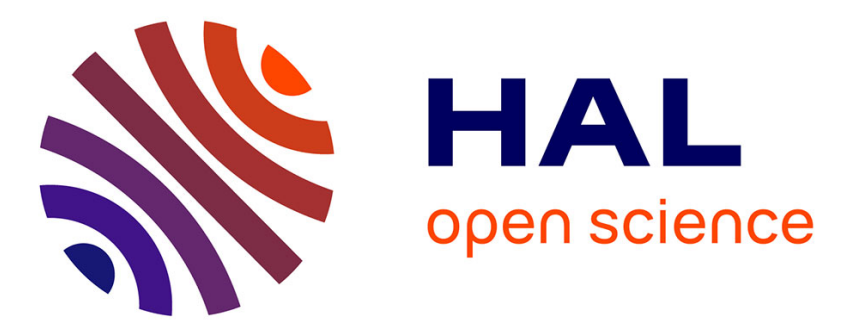

\title{
On the role of bulk viscosity in compressible reactive shear layer developments
}

\author{
Radouan Boukharfane, Pedro José Martínez Ferrer, Arnaud Mura, Vincent
}

Giovangigli

\section{- To cite this version:}

Radouan Boukharfane, Pedro José Martínez Ferrer, Arnaud Mura, Vincent Giovangigli. On the role of bulk viscosity in compressible reactive shear layer developments. European Journal of Mechanics B/Fluids, 2019, 77, pp.32-47. 10.1016/j.euromechflu.2019.02.005 . hal-02059568

\section{HAL Id: hal-02059568 https://hal.science/hal-02059568}

Submitted on 6 Mar 2019

HAL is a multi-disciplinary open access archive for the deposit and dissemination of scientific research documents, whether they are published or not. The documents may come from teaching and research institutions in France or abroad, or from public or private research centers.
L'archive ouverte pluridisciplinaire HAL, est destinée au dépôt et à la diffusion de documents scientifiques de niveau recherche, publiés ou non, émanant des établissements d'enseignement et de recherche français ou étrangers, des laboratoires publics ou privés. 


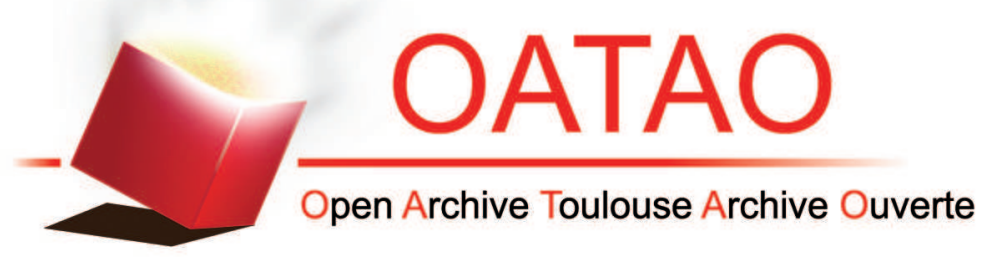

\section{Open Archive Toulouse Archive Ouverte (OATAO)}

OATAO is an open access repository that collects the work of some Toulouse researchers and makes it freely available over the web where possible.

This is an author's version published in: https://oatao.univ-toulouse.fr/22758

Official URL: https://doi.org/10.1016/j.euromechflu.2019.02.005

\section{To cite this version :}

Boukharfane, Radouan and Martínez Ferrer, Pedro José and Mura, Arnaud and Giovangigli, Vincent On the role of bulk viscosity in compressible reactive shear layer developments. (2019) European Journal of Mechanics - B/Fluids, 77. 32-47. ISSN 0997-7546

Any correspondence concerning this service should be sent to the repository administrator: tech-oatao@listes-diff.inp-toulouse.fr 


\title{
On the role of bulk viscosity in compressible reactive shear layer developments
}

\author{
Radouan Boukharfane ${ }^{\mathrm{a}, \mathrm{b}}$, Pedro José Martínez Ferrer ${ }^{\mathrm{a}, \mathrm{c}}$, Arnaud Mura ${ }^{\mathrm{a}, *}$, \\ Vincent Giovangigli ${ }^{\mathrm{d}}$ \\ a PPRIME UPR 3346 CNRS, ENSMA, 86961, Futuroscope Chasseneuil Cedex, France \\ ${ }^{\mathrm{b}}$ ISAE-Supaero, BP 54032, 31055 Toulouse Cedex 04, France \\ c Barcelona Supercomputing Center, C/ Jordi Girona, 29, Barcelona, 08034, Spain \\ d CMAP UMR 7641 CNRS, Ecole Polytechnique, 91128 Palaiseau Cedex, France
}

Keywords:

Bulk viscosity

Shear layer

Direct numerical simulation

Molecular transport

\begin{abstract}
A B S T R A C T
Despite 150 years of research after the reference work of Stokes, it should be acknowledged that some confusion still remains in the literature regarding the importance of bulk viscosity effects in flows of both academic and practical interests. On the one hand, it can be readily shown that the neglection of bulk viscosity (i.e., $\kappa=0$ ) is strictly exact for mono-atomic gases. The corresponding bulk viscosity effects are also unlikely to alter the flowfield dynamics provided that the ratio of the shear viscosity $\mu$ to the bulk viscosity $\kappa$ remains sufficiently large. On the other hand, for polyatomic gases, the scattered available experimental and numerical data show that it is certainly not zero and actually often far from negligible. Therefore, since the ratio $\kappa / \mu$ can display significant variations and may reach very large values (it can exceed thirty for dihydrogen), it remains unclear to what extent the neglection of $\kappa$ holds. The purpose of the present study is thus to analyze the mechanisms through which bulk viscosity and associated processes may alter a canonical turbulent flow. In this context, we perform direct numerical simulations (DNS) of spatially-developing compressible non-reactive and reactive hydrogen-air shear layers interacting with an oblique shock wave. The corresponding flowfield is of special interest for various reactive high-speed flow applications, e.g., scramjets. The corresponding computations either neglect the influence of bulk viscosity $(\kappa=0)$ or take it into consideration by evaluating its value using the EGlib library. The qualitative inspection of the results obtained for two-dimensional cases in either the presence or the absence of bulk viscosity effects shows that the local and instantaneous structure of the mixing layer may be deeply altered when taking bulk viscosity into account. This contrasts with some mean statistical quantities, e.g., the vorticity thickness growth rate, which do not exhibit any significant sensitivity to the bulk viscosity. Enstrophy, Reynolds stress components, and turbulent kinetic energy (TKE) budgets are then evaluated from three-dimensional reactive simulations. Slight modifications are put into evidence on the energy transfer and dissipation contributions. From the obtained results, one may expect that refined large-eddy simulations (LES) may be rather sensitive to the consideration of bulk viscosity, while Reynolds-averaged Navier-Stokes (RANS) simulations, which are based on statistical averages, are not.
\end{abstract}

\section{Introduction}

The bulk (or volume) viscosity $\kappa$, which can be related to the second (or dilatational) viscosity coefficient $\lambda$, is associated to the vibrational and rotational energy of the molecules. From the macroscopic viewpoint, it characterizes the resistance to dilatation of an infinitesimal bulk element at constant shape [1]. It is strictly zero only for dilute monoatomic gases and this theoretical result is often used to discard it, regardless of the nature or internal

\footnotetext{
* Corresponding author.

E-mail address: arnaud.mura@ensma.fr (A. Mura).
}

structure of the fluid as well as the flowfield conditions. However, acoustic absorption measurements performed at room temperature have shown that the ratio of the volume to the shear viscosity $\kappa / \mu$ may be up to thirty for hydrogen at room temperature [2], and recent analyses of reactive multicomponent high-speed flows have confirmed that it is not justified to neglect it, except for the sake of simplicity [3]. The dilatational viscosity is important in describing sound attenuation in gaseous media, and the absorption of sound energy into the fluid depends itself on the sound frequency, i.e., the rate of fluid expansion and compression. For polyatomic gases, the available measurements of $\kappa$, which remains quite seldom due to the complexity of its determination, show that it is certainly not zero and actually far from negligible. It is also noteworthy 
that theoretical analyses do show that $\kappa / \eta$ is at least of the order of unity. Therefore, since the ratio $\kappa / \mu$ can display significant variations and may reach very large values, it is unclear to what extent the Stokes hypothesis (i.e., $\lambda=-2 \mu / 3$ or $\kappa=0$ ) may be used for compressible and turbulent flows of gases featuring a ratio $\kappa / \mu$ greater than unity.

In either an expansion or a contraction of the gas mixture, the work done by the pressure modifies immediately the translational energy of the molecules, while a certain time-lag is needed for the translational and internal energy to re-equilibrate through inelastic collisions [4]. This can be described through a system of two coupled partial differential equations written for the internal and translational temperatures, with a pressure-dilatation term that acts as a source term in the translational temperature budget. The volume (or bulk) viscosity is associated to this relaxation phenomenon and it is evaluated from this internal energy relaxation time-lag. The evaluation of this property for a mixture of polyatomic gases is far from being an easy task since the kinetic theory of gases does not yield an explicit expression for this transport coefficient, but instead linear systems that must be solved [5]. The corresponding systems are derived from polynomial expansions of the species' perturbed distribution functions. The bulk viscosity is obtained here using the library EGlib developed by Ern and Giovangigli [6,7]. It is evaluated as a linear combination of the pure species volume viscosities, which require the evaluation of various collision integrals [5].

The impact of bulk viscosity effects has been previously analyzed in several situations including shock-hydrogen bubble interactions [3], turbulent flames [8], compressible boundary layers [9], shock-boundary layer interaction [10], and planar shockwave [11]. All these studies confirm that the bulk viscosity effects may be significant. The purpose of the present work is to assess its influence in regard to both the instantaneous and statistical features of canonical compressible turbulent multicomponent flows. Using direct numerical simulation (DNS), we investigate the impact of the bulk viscosity coefficient $\kappa$ on the spatial development of reactive and non-reactive compressible mixing layers interacting with an oblique shock wave. Such a canonical flowfield is typical of the shock-mixing layer interactions that take place in compressible flows of practical interest [12]. For instance, supersonic jets at high nozzle-pressure ratio (NPR) give rise to complex cellular structures, where shocks and expansions waves interact with the turbulent outer shear layer [13]. It is also relevant to scramjet intakes and combustors, where shock waves interact with the shear layers issued from the injection systems. On the one hand, it is clear that the occurrence of shock waves in supersonic combustors induces pressure losses that cannot be avoided but, on the other hand, the resulting shock interactions with mixing layers contribute to scalar dissipation (i.e., mixing) rates enhancement [14], and may favor combustion stabilization in high-speed flows.

The present manuscript is organized as follows: the mathematical model is presented in the next section (i.e., Section 2), which also includes a short description of the numerical methods. The details of the computational setup are subsequently provided in Section 3. Section 4 gathers all the results issued from (i) twodimensional numerical simulations of both inert (4.1) and reactive (4.2) cases, and (ii) the three-dimensional case, which is analyzed in 4.3. Finally, some concluding remarks and perspectives for future works are presented in Section 5.

\section{Mathematical description and computational model}

In this work, the in-house massively parallel DNS solver CREAMS is used. It solves the unsteady, three-dimensional set of compressible Navier-Stokes equations for multicomponent reactive mixtures [15]:

$\partial_{t}(\rho)+\nabla \cdot(\rho \boldsymbol{u})=0$, $\partial_{t}(\rho \boldsymbol{u})+\nabla \cdot(\rho \boldsymbol{u} \otimes \boldsymbol{u})=\nabla \cdot \boldsymbol{\sigma}$,

$\partial_{t}\left(\rho \mathcal{E}_{t}\right)+\nabla \cdot\left(\rho \boldsymbol{u} \mathcal{E}_{t}\right)=\nabla \cdot(\sigma \cdot \boldsymbol{u}-\mathcal{J})$,

$\partial_{t}\left(\rho Y_{\alpha}\right)+\nabla \cdot\left(\rho \boldsymbol{u} Y_{\alpha}\right)=-\nabla \cdot\left(\rho \boldsymbol{V}_{\alpha} Y_{\alpha}\right)+\rho \dot{\omega}_{\alpha}$,

where $t$ denotes the time, $\boldsymbol{\nabla}$ is the spatial derivative operator, $\boldsymbol{u}$ is the flow velocity, $\rho$ is the density, $\mathcal{E}_{t}=e+\boldsymbol{u} \cdot \boldsymbol{u} / 2$ is the total specific energy (obtained as the sum of the internal specific energy, $e$, and kinetic energy), $Y_{\alpha}$ is the mass fraction of chemical species $\alpha$ (with $\left.\alpha \in \mathscr{S}=\left\{1, \ldots, \mathcal{N}_{\mathrm{sp}}\right\}\right), \boldsymbol{V}_{\alpha}$ is the diffusion velocity of species $\alpha, \mathcal{J}$ is the heat flux vector and $\dot{\omega}_{\alpha}$ represents the chemical production rate of species $\alpha$. The integer $\mathcal{N}_{\mathrm{sp}}$ denotes the number of chemical species.

The above set of conservation equations (1) requires to be completed by constitutive laws. In this respect, the ideal gas mixture equation of state (EoS), $P=\rho \mathcal{R} T / \mathcal{W}$ with $\mathcal{R}$ the universal gas constant, is used to relate the pressure $P$ to the temperature $T$. In this expression, the quantity $\mathcal{W}$ denotes the molar weight of the multicomponent mixture, which is obtained as the sum of the molecular mass of each individual species $\mathcal{W}^{-1}=\sum_{\alpha=1}^{\mathcal{N}_{\mathrm{sp}}} Y_{\alpha} / \mathcal{W}_{\alpha}$. Within the framework of the kinetic theory of dilute polyatomic gas mixtures, the molecular diffusion velocity vector $\boldsymbol{V}_{\alpha}, \alpha \in \mathscr{S}$, heat flux vector $\mathcal{J}$, and second-order stress tensor $\sigma$ are expressed as follows:

$\rho \boldsymbol{V}_{\alpha} Y_{\alpha}=-\sum_{\beta \in \mathscr{S}} \rho Y_{\alpha} \mathscr{D}_{\alpha, \beta}\left(\boldsymbol{d}_{\beta}+\chi_{\beta} X_{\beta} \nabla(\ln T)\right)$,

$\mathcal{J}=\sum_{\alpha \in \mathscr{S}} \rho \boldsymbol{V}_{\alpha} Y_{\alpha}\left(h_{\alpha}+\frac{\mathcal{R} T \chi_{\alpha}}{\mathcal{W}_{\alpha}}\right)-\lambda_{T} \nabla T$

$\sigma=-P \boldsymbol{I}+\boldsymbol{\tau}=-P \boldsymbol{I}+\mu\left(\nabla \boldsymbol{u}+\nabla \boldsymbol{u}^{\top}\right)+\lambda(\nabla \cdot \boldsymbol{u}) \boldsymbol{I}$,

where $\mathscr{D}_{\alpha, \beta}$ are the multicomponent diffusion coefficients, $\boldsymbol{d}_{\alpha}, \alpha \in$ $\mathscr{S}$, the species diffusion driving forces, $\chi_{\alpha}$ the rescaled thermal diffusion ratios, $X_{\alpha}$ the species mole fractions, $h_{\alpha}$ the enthalpy per unit mass of the $\alpha$ th species, and $\lambda_{T}$ the thermal conductivity. The diffusion driving force $\boldsymbol{d}_{\alpha}$ of the $\alpha$ th species is given by $\boldsymbol{d}_{\alpha}=\nabla X_{\alpha}+$ $\left(X_{\alpha}-Y_{\alpha}\right) \nabla(\ln P)$. The quantity $\mu$ denotes the shear viscosity and $\lambda$ denotes the second (or dilatation) viscosity coefficient.

The bulk viscosity coefficient $\kappa$ appears explicitly in the expression of the viscous stress tensor $\boldsymbol{\tau}$. A relationship between the bulk viscosity $\kappa$ and viscosity coefficients $\mu$ and $\lambda$ can be deduced from the expression of the total pressure, which can be evaluated as the component of the spherical tensor based on the trace of the total stress tensor $\sigma$ :

$$
-\frac{\operatorname{tr}(\sigma)}{3}=-\sum_{i=1}^{i=3} \frac{\sigma_{i i}}{3}=P-\left(\lambda+\frac{2}{3} \mu\right) \nabla \cdot \boldsymbol{u}=P-\kappa \nabla \cdot \boldsymbol{u}
$$

The second term in the right-hand-side of the above expression is the dilational contribution, which defines the bulk viscosity as $\kappa=$ $\lambda+2 \mu / 3$. As mentioned above, the Stokes' hypothesis, stating that $\lambda=-2 \mu / 3$ (and hence $\kappa=0$ ), is often retained as a simplifying assumption. Many efforts have been devoted to the derivation of relationships between the bulk viscosity and fundamental fluid properties [16,17]. If we consider a single polyatomic gas with a unique internal energy mode, the internal energy relaxation time $\tau^{\text {int }}$ can be related to the bulk viscosity $[4,18]$ :

$\kappa=\left(P \mathcal{R} / c_{v}^{2}\right) \cdot c^{\text {int }} \tau^{\text {int }}$,

when the relaxation time is smaller than fluid characteristic times. In the above expression, $c^{\text {int }}$ denotes the internal heat capacity and $c_{v}$ the specific heat at constant volume. When there are several internal energy modes and/or several species present in the mixture, 
the above simple expression is replaced by the solution to a linear system [19]. Within the Monchick and Mason approximation [20], neglecting complex collisions characterized by more than one quantum jump, the reduced system is diagonal and yields $\kappa$ [3]:

$\kappa=\left(P \mathcal{R} / c_{v}^{2}\right) \cdot \sum_{k \in \mathfrak{P}} X_{k} c_{k}^{\text {int }} \tau_{k}^{\text {int }}$,

where the integer $\mathfrak{P}=1, \ldots, n^{p}$ is the polyatomic species indexing set. The average relaxation time for internal energy of the $k$ th species $\tau_{k}^{\text {int }}$ is then expressed as:

$c_{k}^{\text {int }} / \tau_{k}^{\text {int }}=\sum_{l \in \mathfrak{N}} c_{k}^{l} / \tau_{k}^{l}$,

where $\tau_{k}^{l}$ denotes the average relaxation time of internal energy mode $l$ for the $k$ th species, and $\mathfrak{N}$ is the internal energy mode indexing set.

The CREAMS solver is coupled with the EGlib library to estimate transport coefficients from the kinetic theory of gases [21]. In this library, the optimized subroutines EGSKm are used to evaluate the bulk viscosity. The integer $m \in \llbracket 2,6 \rrbracket$ associated to the subroutine name refers to retained level of approximation. The higher the value of $m$, the more expensive the algorithm but also the more accurate the bulk viscosity expression. Following the work of Billet et al. [3], the value $m=3$ is retained for the purpose of the present study. The shear viscosity and diffusion velocities are evaluated with the routines EGFE3 and EGFYV, respectively. EGFLCT3 is used to determine the thermal conductivity $\lambda_{T}$ and rescaled thermal diffusion ratios $\chi_{\alpha}$. In this respect, some additional computations showed that the extra time needed for $m=4$ and $m=5$ is approximately $30 \%$ in comparison with the one needed for $m=2$ and $m=3$, while an additional time larger than $250 \%$ is required for $m=6$ compared to $m=2$ and $m=3$.

The above system (1) is discretized on a Cartesian grid. A seventh-order accurate WENO scheme is used to approximate inviscid fluxes, while an eighth-order accurate centered difference scheme is retained to approximate viscous and diffusive contributions. Time integration is performed with a third-order accurate TVD Runge-Kutta scheme. The stiffness associated to the wide range of time scales involved in the description of the chemical system is addressed using the Sundials CVODE solver [22]. A standard splitting operator technique, similar to the one previously retained in Ref. [23], is used. A detailed verification of the solver may be found in Refs. [15,24].

\section{Problem statement and computational setup}

We study the interaction of an oblique shock with a spatiallydeveloping shear layer. The upper stream corresponds to the fuel inlet, i.e., a mixture containing hydrogen, and the bottom inlet stream to vitiated air. Both two- and three-dimensional computations are performed. Fig. 1 provides a typical sketch of the corresponding computational geometry and Table 1 gathers the values of the main parameters relevant to the present numerical simulation. The flow initialization is similar to the one retained in Ref. [15]. Assuming equal free-stream specific heat capacity ratios, the convective Mach number may be evaluated from $M_{c}=\left(U_{1}-\right.$ $\left.\mathrm{U}_{2}\right) /\left(a_{1}+a_{2}\right)$, where $a_{1}$ and $a_{2}$ denotes the sonic speeds of streams 1 (oxidizer inlet stream) and 2 (fuel inlet stream) respectively. For the present set of computations, it is equal to $M_{c}=0.48$. The values of the velocity at the inlets are $U_{1}=1634.0 \mathrm{~m} / \mathrm{s}$ at bottom (oxidizer stream) and $U_{2}=973.0 \mathrm{~m} / \mathrm{s}$ at top (fuel stream).

The mixing layer flow is impinged by an oblique shock wave that is issued from the oxidizer inlet stream (1) at the bottom boundary. The oblique shock wave angle is $\beta=33^{\circ}$, see Fig. 1 . The geometrical parameters relevant to the present set of numerical
Table 1

Parameters of the shock-mixing layer interaction case.

\begin{tabular}{llll}
\hline & Fuel & Oxidizer & Bottom \\
\hline$P(\mathrm{~Pa})$ & 94232.25 & 94232.25 & 129951.0 \\
$T(\mathrm{~K})$ & 545.0 & 1475.0 & 1582.6 \\
$u_{1}(\mathrm{~m} / \mathrm{s})$ & 973.0 & 1634.0 & 1526.3 \\
$u_{2}(\mathrm{~m} / \mathrm{s})$ & 0.0 & 0.0 & 165.7 \\
$u_{3}(\mathrm{~m} / \mathrm{s})$ & 0.0 & 0.0 & 0.0 \\
$\mathrm{Mach}(-)$ & 1.6 & 2.12 & 1.93 \\
$Y_{\mathrm{H}_{2}}(-)$ & 0.05 & 0.0 & 0.0 \\
$Y_{\mathrm{O}_{2}}(-)$ & 0.0 & 0.278 & 0.278 \\
$Y_{\mathrm{H}_{2} \mathrm{O}}(-)$ & 0.0 & 0.17 & 0.17 \\
$Y_{\mathrm{H}}(-)$ & 0.0 & $5.60 \cdot 10^{-7}$ & $5.60 \cdot 10^{-7}$ \\
$Y_{\mathrm{O}}(-)$ & 0.0 & $1.55 \cdot 10^{-4}$ & $1.55 \cdot 10^{-4}$ \\
$Y_{\mathrm{OH}}(-)$ & 0.0 & $1.83 \cdot 10^{-3}$ & $1.83 \cdot 10^{-3}$ \\
$Y_{\mathrm{HO}_{2}}(-)$ & 0.0 & $5.10 \times 10^{-6}$ & $5.10 \times 10^{-6}$ \\
$Y_{\mathrm{H}_{2} \mathrm{O}_{2}}(-)$ & 0.0 & $2.50 \times 10^{-7}$ & $2.50 \times 10^{-7}$ \\
$Y_{\mathrm{N}_{2}}(-)$ & 0.95 & 0.55 & 0.55 \\
\hline
\end{tabular}

Table 2

Computational mesh description.

\begin{tabular}{lllllll}
\hline$L_{x_{1}}$ & $L_{x_{2}}$ & $L_{x_{3}}$ & $N_{x_{1}}$ & $N_{x_{2}}$ & $N_{x_{3}}$ & $\delta_{\omega, 0}(\mathrm{~m})$ \\
\hline 280 & 130 & 15 & 1640 & 750 & 180 & $1.44 \times 10^{-4}$ \\
\hline
\end{tabular}

simulations are provided in Table 2. The quantities $L_{x_{1}}, L_{x_{2}}$, and $L_{x_{3}}$ denote the computational domain lengths in each direction normalized by the initial vorticity thickness $\delta_{\omega, 0}$, while $N_{x_{1}}, N_{x_{2}}$, and $N_{x_{3}}$ are the corresponding numbers of grid points. In the two-dimensional computations, only the $x_{1}$ - and $x_{2}$-directions are considered.

The flow is initialized with a hyperbolic tangent profile for the streamwise velocity component, while the other velocity components are set to zero. Species mass fractions and density are also set according to the following general expression:

$\varphi\left(x_{1}, x_{2}, x_{3}\right)=\frac{\varphi_{1}+\varphi_{2}}{2}+\frac{\varphi_{1}-\varphi_{2}}{2} \tanh \left(\frac{2 x_{2}}{\delta_{\omega, 0}}\right)$,

where $\varphi$ denotes any of the flow variables mentioned above (i.e., species mass fraction or streamwise velocity component). The value of the Reynolds number $R e_{\delta_{\omega}}$, based on the initial vorticity thickness and inlet velocity difference $\Delta \mathrm{U}=\mathrm{U}_{1}-\mathrm{U}_{2}$ is $R e_{\delta_{\omega}}=640$. One of the fundamental statistical quantities that characterizes the mixing layer development is its normalized growth rate [25]. Although the definition of this growth rate is not unique, one standard expression relies on the vorticity thickness definition [26,27]:

$\delta_{\omega}\left(x_{1}\right)=\frac{\mathrm{U}_{1}-\mathrm{U}_{2}}{\partial \widetilde{u}_{1} /\left.\partial x_{2}\right|_{\max }}$.

Dirichlet boundary conditions are applied at the two supersonic inlets, perfectly non-reflecting boundary conditions are set at the outflow, and periodic boundary conditions are settled along the $x_{3}-$ direction. A slip boundary condition is imposed at the top, while the bottom boundary condition is set by using Rankine-Hugoniot relations, generalized for a multicomponent mixture [28]. In order to trigger flow transition, a slight white noise fluctuation is superimposed to the transverse velocity component along the line $\left(x_{1}, x_{2}\right)=\left(4 \delta_{\omega, 0}, 0\right)$. The value of the CFL number is set to 0.75 . Reactive flow simulations are conducted with the detailed mechanism of O'Conaire et al. [29]. It consists of nine chemical species $\left(\mathrm{H}_{2}\right.$, $\mathrm{O}_{2}, \mathrm{H}_{2} \mathrm{O}, \mathrm{H}, \mathrm{O}, \mathrm{OH}, \mathrm{HO}_{2}, \mathrm{H}_{2} \mathrm{O}_{2}$, and $\mathrm{N}_{2}$ ) and 21 elementary reaction steps. The concentrations of these species at the inlet have been determined from equilibrium conditions so as to reach favorable self-ignition conditions within the extension of the computational domain.

Throughout this manuscript, the Reynolds and Favre averages of any quantity $\varphi$ are denoted by $\bar{\varphi}$ and $\widetilde{\varphi}$, while the corresponding 


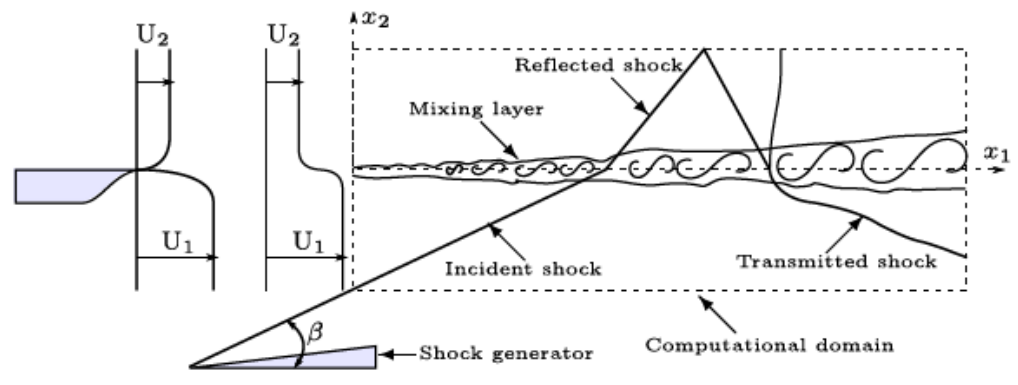

Fig. 1. Sketch of the two-dimensional shock-mixing layer interaction geometry.

statistical fluctuations are denoted by single and double primes, i.e., $\varphi^{\prime}$ and $\varphi^{\prime \prime}$, respectively. Averaging is performed over the direction of statistical homogeneity and time $t$.

As emphasized by Buttay and co-workers [30], it is not obvious to provide a firm and convincing assessment of the computational resolution for transitional (i.e., spatially-developing) flowfields and this may require the consideration of various criteria as previously done in Refs. [13,31]. However, for the present three-dimensional case, the computational grid size $\Delta$ is such that the Kolmogorov length scale, as evaluated from its standard expression $L_{\eta}=$ $\left(v^{3} / \epsilon\right)^{1 / 4}$, is approximately equal to $\Delta$. Therefore, the present set of computational results can be considered as direct numerical simulation (DNS) results. Indeed, the spatial resolution of standard DNS computations is generally set from the following criterion: $k_{\max } \cdot L_{\eta}=3 / 2$, where $k_{\max }=\pi / \Delta$ denotes the maximum value of the wave number, with $\Delta$ the typical mesh grid size [32]. Thus, provided that this criterion is fulfilled, the Kolmogorov length scale $L_{\eta}$ should be larger than $\Delta / 2$, which is presently the case.

\section{Analysis of computational results}

\subsection{Inert two-dimensional mixing layer}

Figure 2 displays the instantaneous field of the ratio $\kappa / \mu$ computed for the present flow conditions. From this figure, it is noteworthy that (i) this ratio reaches values significantly larger than unity, (ii) it exhibits important spatial variations, the most significant of which are related to mixture composition. As expected, it displays no sensitivity to pressure variations (i.e., shock waves). Considering the values of $\kappa / \mu$, as well as the amplitude of its variations, ${ }^{1}$ one may expect some remarkable effects of the bulk viscosity on this inert flowfield. It is the objective of this preliminary section to study to what extent the bulk viscosity may influence the instantaneous and statistical characteristics of the two-dimensional mixing layer development.

Instantaneous flow visualizations are very revealing of some local features of the shear layer, which are filtered out once fields or cross-stream profiles of averaged quantities are considered instead. For instance, quantitative comparisons of the onset of the streamwise vortices formation can be obtained from the instantaneous fields of the dimensionless magnitude of the density gradient, i.e., "numerical Schlieren", reported in Fig. 3.

In this figure, it is remarkable that, in the absence of bulk viscosity, the normalized abscissa at which the vortex roll-up processes take place is approximately $x_{1} / \delta_{\omega, 0}=103.0$, while in the situation featuring non-zero value of the bulk viscosity, it can be estimated to be $x_{1} / \delta_{\omega, 0}=116.0$. It should be emphasized here that only one typical set of instantaneous snapshots is reported in the present manuscript just for the sake of conciseness, but the corresponding vortex roll-up process is systematically observed to take place

\footnotetext{
1 It is noteworthy that, as a consequence of fuel dilution with nitrogen, the values of this ratio remain smaller than those obtained for pure hydrogen.
}

early in the absence of volume viscosity. This can be explained by the same argument as the one invoked by Billet et al. [3] in their study of shock/hydrogen bubble interaction. The shear layer is also a diffusion layer associated to a density gradient, the absence of bulk viscosity makes the baroclinic term $\nabla P \times \nabla \rho / \rho^{2}$ greater at the shock/mixing layer interaction location, thus favoring the birth of velocity fluctuations. When volume viscosity is taken into account, the shock is much smoother in agreement with the physical theory of shock wave internal structure. As a consequence, the baroclinic production term is lower than in absence of volume viscosity. The interaction between the mixing layer and the shock wave can be further assessed by considering the Richtmyer-Meshkov instability development, the key point of which is the baroclinic effect [33]. The Richtmyer-Meshkov instability indeed takes place when two fluids having different densities are impulsively accelerated, similarly to what occurs when the shock wave impinges the mixing layer in the present study. This process may be analyzed by considering the transport equation for the enstrophy $\Omega=|\omega|^{2} / 2$ :

$$
\begin{aligned}
\frac{\mathrm{D} \Omega}{\mathrm{D} t} & =\partial_{t} \Omega+\boldsymbol{u} \cdot \nabla \Omega=\underbrace{\frac{1}{2} \boldsymbol{\omega} \cdot\left(\nabla \boldsymbol{u}+\nabla \boldsymbol{u}^{\top}\right) \cdot \omega}_{\mathcal{E}}-\underbrace{\Omega \boldsymbol{\Omega} \cdot \boldsymbol{u}}_{\mathcal{D}} \\
& +\underbrace{\omega \cdot\left(\frac{\nabla P \times \nabla \rho}{\rho^{2}}\right)}_{\mathcal{B}}+\underbrace{\omega \cdot\left(\nabla \times\left[\frac{\nabla \cdot \boldsymbol{\tau}}{\rho}\right]\right)}_{\mathcal{V}}
\end{aligned}
$$

The production/destruction terms on the right hand side (RHS) of (9) are associated to vortex-stretching, dilatation, baroclinic torque, and viscous dissipation. The baroclinic contribution $\nabla P \times$ $\nabla \rho$ plays a significant role in the enstrophy and vorticity production and it appears as one of the main sources of vorticity in supersonic flows [34].

Figure 4 displays the instantaneous field of the magnitude of $\nabla P \times \nabla \rho$ within a region restricted to mixture fraction values such that $\xi \in[0.05,0.95]$. This passive scalar $\xi$ is defined on the basis of conserved elemental (i.e., atomic) mass fractions [35]. The mass fraction of chemical element $\gamma$, denoted $a_{\gamma}$, is readily deduced from the chemical species mass fractions:

$a_{\gamma}=\sum_{\alpha=1}^{\mathcal{N} \text { sp }} \frac{Y_{\alpha} N_{\alpha, \gamma} A_{\gamma}}{W_{\alpha}}$,

where $A_{\gamma}$ is the atomic weight associated to the element $\gamma$ and $N_{\alpha, \gamma}$ denotes the number of $\gamma$ atoms present in each molecule of chemical species $\alpha$. For a two-feeding inlet system such as the one considered here, ${ }^{2}$ the mixture fraction is then obtained by summing over all elemental mass fractions and normalizing the result:

$\xi=\frac{\sum_{\gamma}\left|a_{\gamma}-a_{\gamma, \mathrm{o}}\right|}{\sum_{\gamma}\left|a_{\gamma, \mathrm{F}}-a_{\gamma, \mathrm{o}}\right|}$,

2 Situations featuring more than two inlets were recently addressed by Gomet et al. [36] 


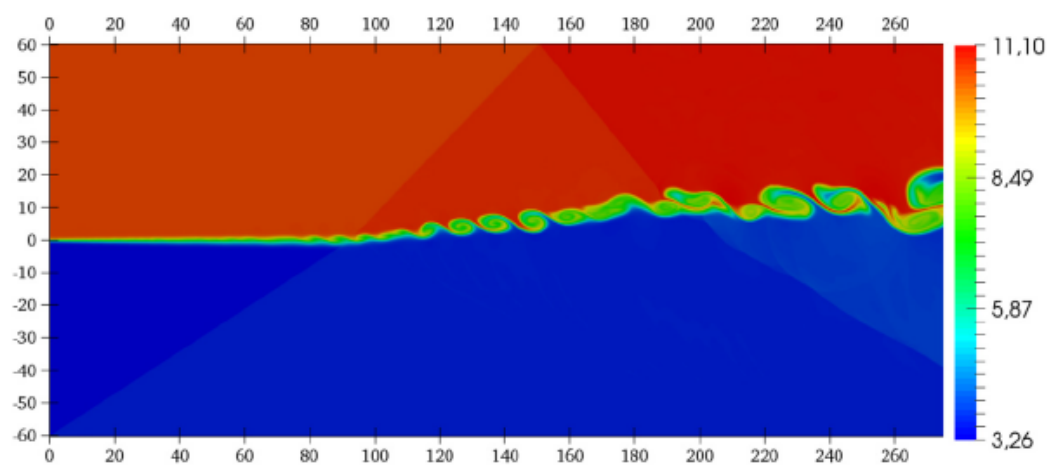

Fig. 2. Instantaneous field of the ratio $\kappa / \mu$ in the case $\kappa \neq 0$ at $t \Delta \mathrm{U} / \delta_{\omega, 0}=75.0$.

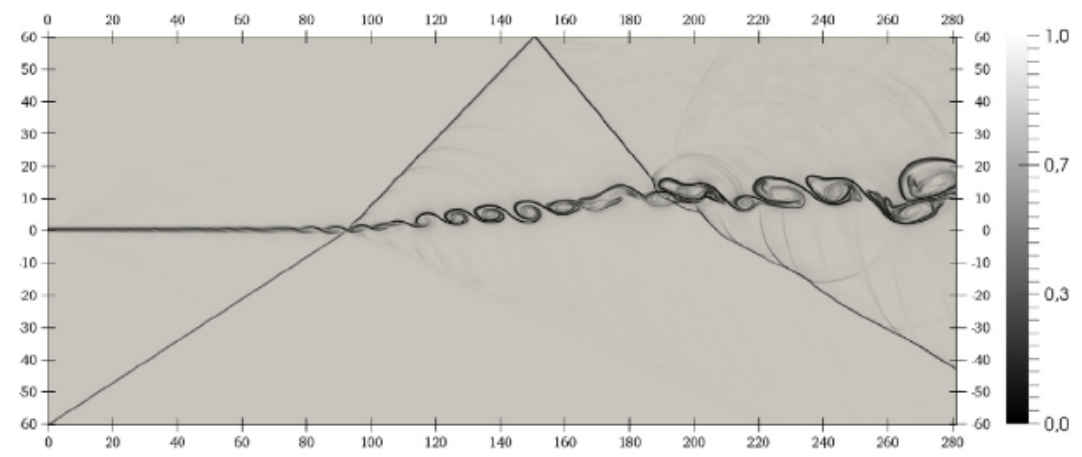

(a) $\kappa \neq 0$

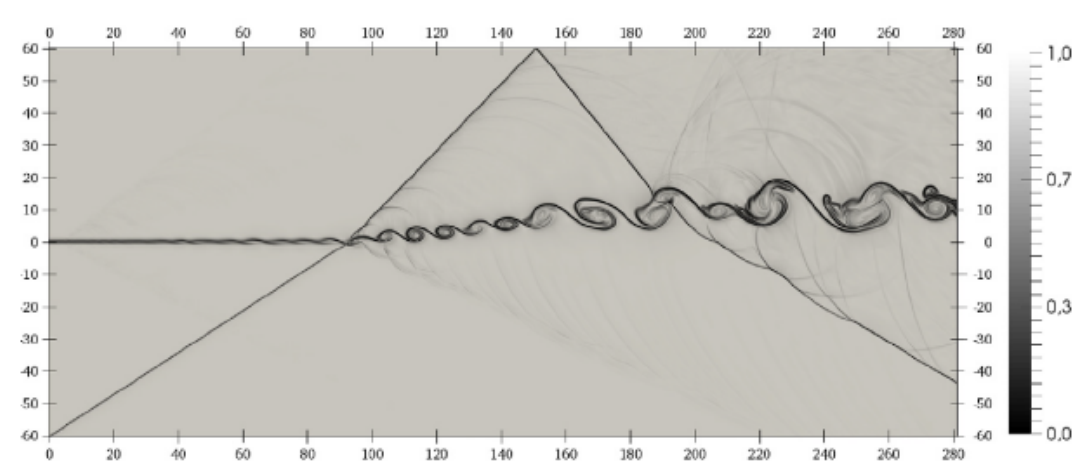

(b) $\kappa=0$

Fig. 3. Instantaneous field of the numerical density-based Schlieren at $t \Delta \mathrm{U} / \delta_{\omega, 0}=75.0$.

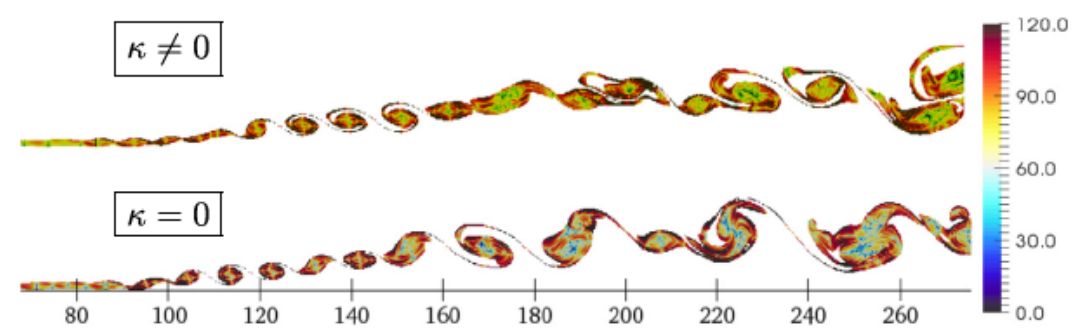

Fig. 4. Instantaneous field of the mixing zone colored by $\|\nabla P \times \nabla \rho\|$.

where $a_{\gamma, 0}$ and $a_{\gamma, \mathrm{F}}$ denote the mass fractions of atom $\gamma$ in the oxidizer and fuel inlet streams, respectively.

Figure 4 shows that the term $\nabla P \times \nabla \rho$ displays large values at the edges of the mixing layer where the pressure gradient and the density gradient are significantly misaligned, thus promoting the development of the mixing layer. The misalignment of the pressure gradient - that is imposed by the shock wave - and local density gradient - that is associated with the mixing layer - serves as a basis to vorticity generation through the baroclinic term. It may contribute significantly to mixing enhancement.

To quantify the vorticity production that is induced by the baroclinic term in the presence or in the absence of bulk viscosity, 

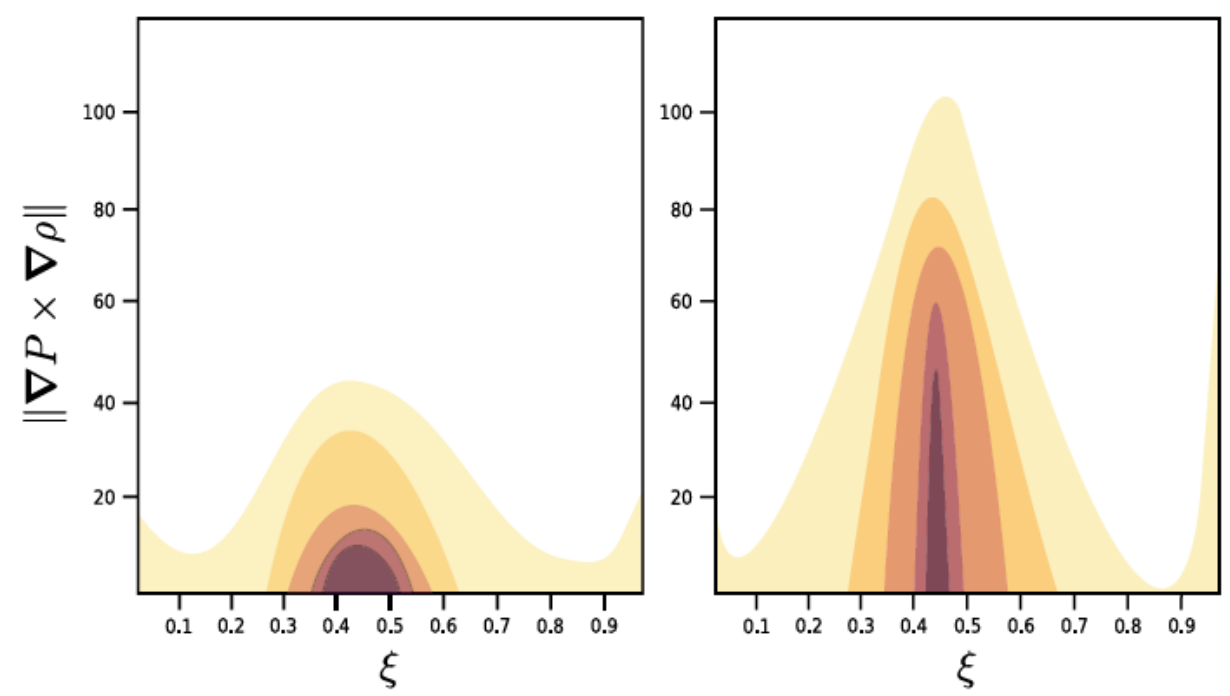

Fig. 5. JPDF of $\xi$ and $\|\nabla P \times \nabla \rho\|$ for $\kappa \neq 0$ (left) and $\kappa=0$ (right).

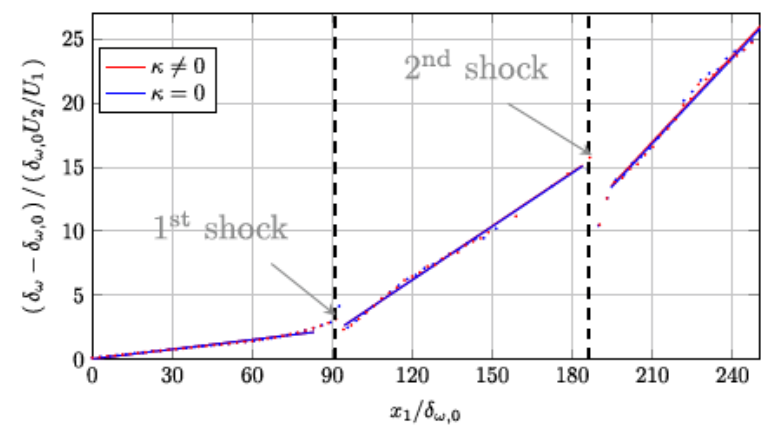

Fig. 6. Two-dimensional inert simulation: spatial evolution of the normalized vorticity thickness.

Fig. 5 reports the joint probability density function (JPDF) of the passive scalar $\xi$ and baroclinic term $\|\nabla P \times \nabla \rho\|$ obtained for both cases. This figure reveals that the production of vorticity by baroclinic effects is concentrated around the stoichiometric mixture fraction value $\xi_{\text {st }}=0.43$ and is dramatically different in the presence of bulk viscosity. The neglection of bulk viscosity tends to enhance artificially large values of this production term.

Figure 6 displays the spatial evolution of normalized vorticity thickness for both cases. It reveals that (i) the interaction of the reflected shock wave with the mixing zone changes significantly the mixing layer growth rate (i.e., the slope) and (ii) the evolution of the mixing layer displays three distinct zones. In the first zone (on the left), before the interaction of the incident shock with the shear layer, there is a quasi-linear growth of the mixing layer thickness. After the shock interaction, there is a significant increase in the mixing layer growth rate in the second zone (i.e., in the middle of the graph). A similar behavior is observed in the third region (on the right) after the interaction of the reflected shock wave with the mixing layer. Finally, the inert case does not exhibit any noticeable difference with respect to the effect of the bulk viscosity: the profiles of the longitudinal evolution of the vorticity thickness for the cases $\kappa \neq 0$ and $\kappa=0$ are indeed almost superimposed.

Attention is now focused on the spatial evolution of the normalized turbulent kinetic energy $\bar{\rho} \mathcal{K} /\left(\rho_{0} \Delta \mathrm{U}^{2}\right)$ with $\mathcal{K}=\widehat{u_{i}^{\prime \prime} u_{i}^{\prime \prime}}$. More specifically, special attention is paid to the maximum in the cross-stream profile of $\mathcal{K}$, which is reported in Fig. 7(a). The comparison performed between both cases $(\kappa=0$ and $\kappa \neq 0$ ) confirms that the molecular bulk viscosity only slightly affects

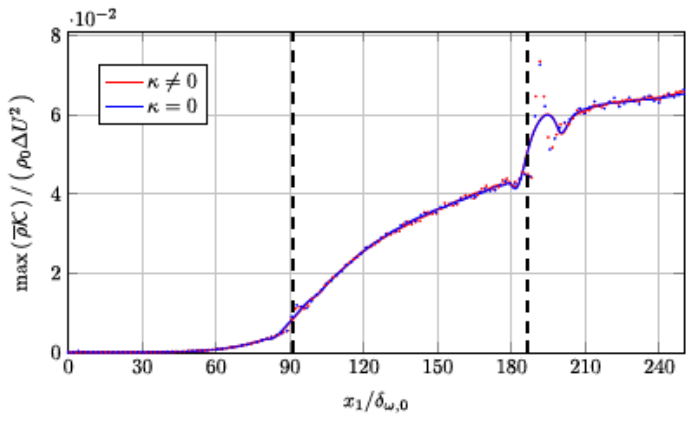

(a)

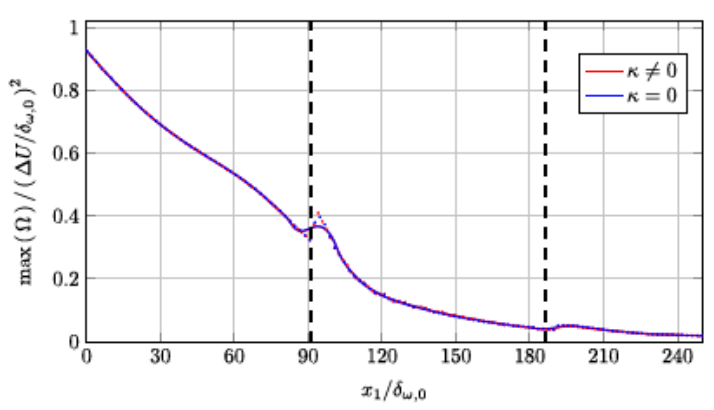

(b)

Fig. 7. Inert simulation: spatial evolution of the longitudinal maxima of (a) normalized turbulent kinetic energy $\bar{\rho} \mathcal{K}$ and (b) normalized enstrophy $\Omega$.

the average development of turbulent kinetic energy in the inert case. Finally, Fig. 7(b) displays the longitudinal evolution of the maximum enstrophy value $\Omega$ normalized by $\left(\Delta \mathrm{U} / \delta_{\omega, 0}\right)^{2}$. As shown in this figure, enstrophy decreases continuously as the mixing layer develops, whether or not the bulk viscosity is taken into account. The enstrophy is also found to be quite slightly impacted by the bulk viscosity for the present set of two-dimensional nonreactive flow computations. Thus, it is concluded that, although the instantaneous fields of vorticity display significant differences depending on the consideration (or not) of the bulk viscosity, the time-averaged statistics tends to dwindle these differences, which are no longer visible on mean enstrophy and turbulent kinetic energy profiles. 


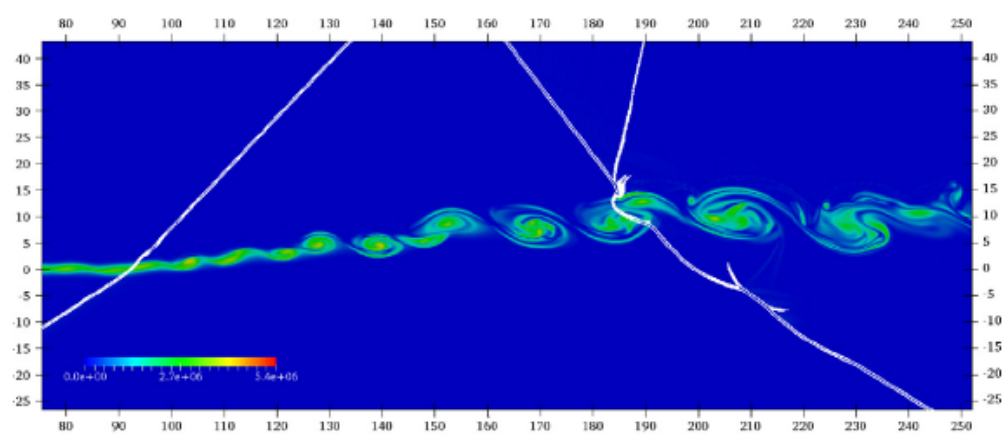

(a) $\kappa \neq 0$

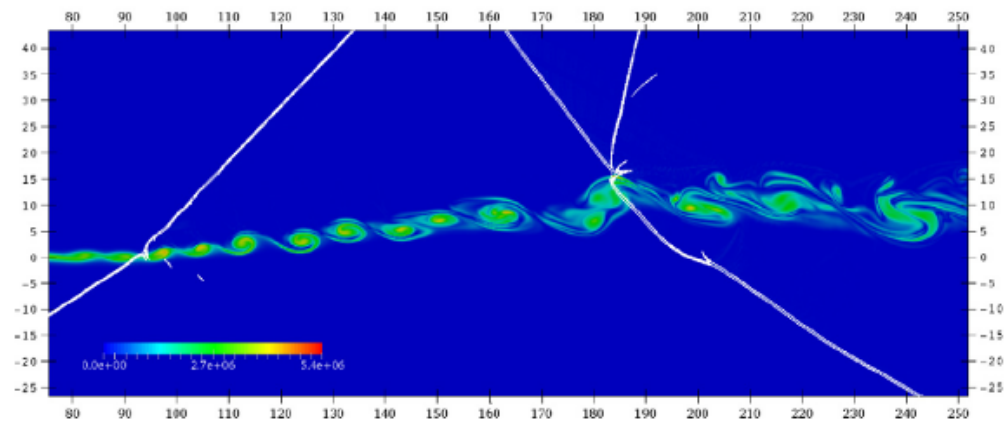

(b) $\kappa=0$

Fig. 8. Instantaneous field of vorticity superimposed with iso-lines of the pressure gradient (in white).

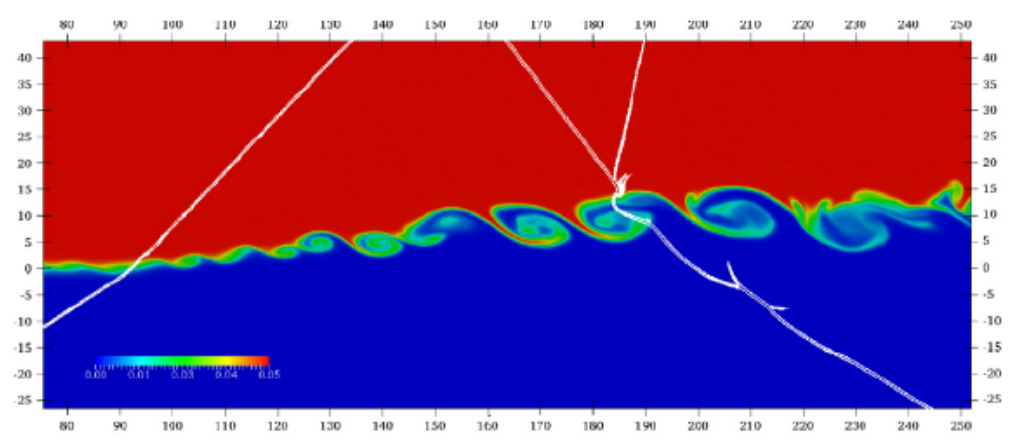

(a) $\kappa \neq 0$

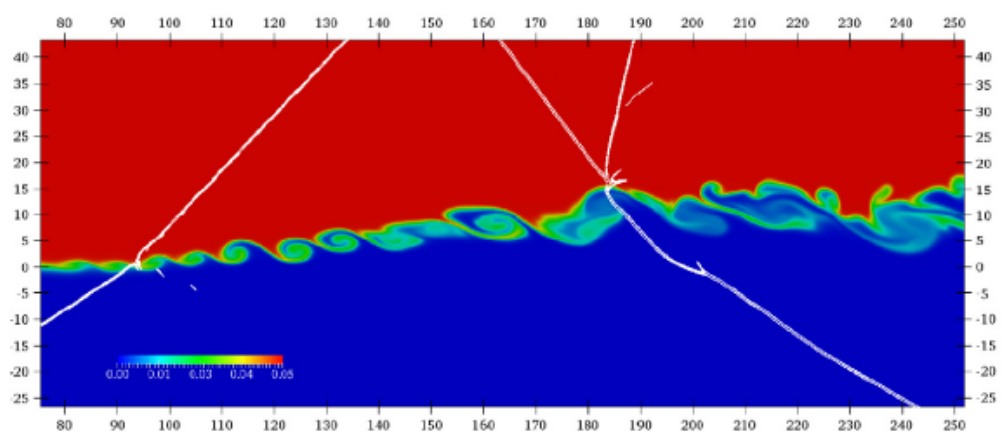

(b) $\kappa=0$

Fig. 9. Instantaneous field of $\mathrm{H}_{2}$ mass fraction superimposed with iso-lines of the pressure gradient (in white). 


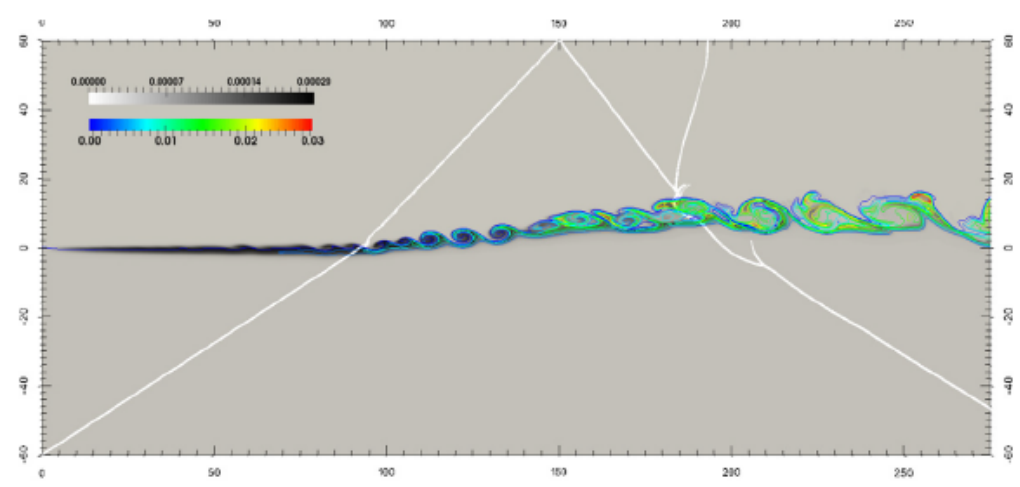

(a) $\kappa \neq 0$

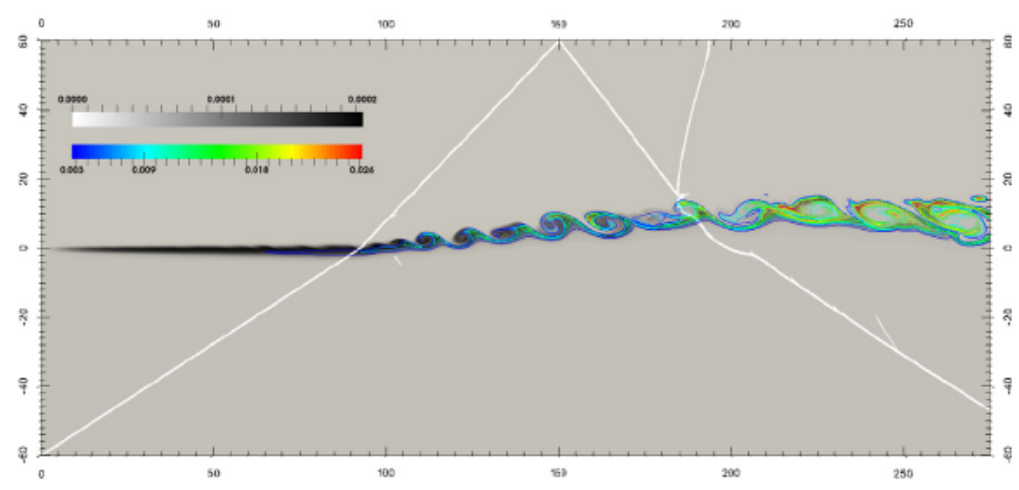

(b) $\kappa=0$

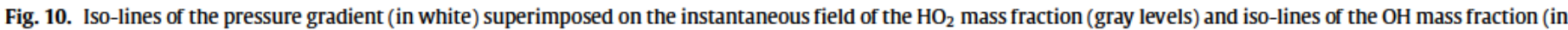
color).

\subsection{Reactive two-dimensional mixing layer}

The analysis of two-dimensional inert mixing layer developments showed that the bulk viscosity only appreciably affects the instantaneous flowfields. Indeed, whether the bulk viscosity is considered or not, the statistical quantities that characterize the mixing layer development exhibit almost no difference. Since the bulk viscosity may affect only the smallest scales, i.e., the molecular ones at which chemical reactions take place, reactive cases may display more significant differences than those observed in inert cases. Therefore, reactive shear layer computations are carried out including (or not) the effects of the bulk viscosity.

For instance, Fig. 8 reports the instantaneous vorticity field obtained for the two conditions ( $\kappa=0$ and $\kappa \neq 0$ ) taken at the same physical time. Iso-lines of the pressure gradient are superimposed on the vorticity field. In contrast to the case without bulk viscosity effect (see Fig. 8(b)), when bulk viscosity effects are taken into account (see Fig. 8(a)), the flow topology remains an ordered whole and the morphology of the vortices retains its coherence after the second interaction with the shock. Moreover, in the absence of bulk viscosity effects, one can notice a premature pairing of vortices similar to the one previously observed in inert cases. The abscissae at which this occurs are $x_{1} / \delta_{\omega, 0} \approx 110$ for $\kappa=0$ and $x_{1} / \delta_{\omega, 0} \approx 127$ for $\kappa \neq 0$, respectively. Figure 9 displays the instantaneous field of the hydrogen mass fraction together with the iso-lines of the pressure gradient. This figure shows that the bulk viscosity contributes to maintain a greater flow coherence, which favors the identification of large roll-ups. In the absence of bulk viscosity effects, the vortex structures issued from the reflected shock interaction are indeed much more disorganized and elongated.
The auto-ignition process is now discussed in the light of Figs. 10 and 11. The first figure reports the hydroperoxyl and hydroxyl radical mass fractions superimposed with isolines of the norm of the pressure gradient. It is noteworthy that $\mathrm{HO}_{2}$ formation begins just downstream of the injection plane in the middle of the mixing layer. Moreover, further downstream begins an induction process with a small amount of these $\mathrm{HO}_{2}$ radicals converted into $\mathrm{OH}$ radicals in the fuel-lean mixture before the shock wave impingement zone. The production of these $\mathrm{OH}$ radicals increases significantly with the formation of pools in the kernels of the vortices while the concentration of $\mathrm{HO}_{2}$ radicals progressively vanishes. These radical pools coincide with the high temperature regions discussed above, allowing for the thermal runaway of the reactive processes. The maximum levels of hydroxyl radicals are found after the reflected shock interacts with the mixing layer, where the heat release rate reaches its maximum value. The remaining hydroperoxyl radicals concentrate below the fuel-lean mixture region, on the oxidizer side. The bulk viscosity has the effect of increasing the maximum level of hydroxyl radical concentration, as well as the heat release rate. The latter decreases significantly when the mixing layer interacts with the second (i.e., reflected) shock wave. These different observations highlight the effect of the bulk viscosity on the instantaneous development of the reactive mixing layer, an effect which is quite remarkable. This confirms the observations made in the inert case.

The modifications that can be induced by the bulk viscosity effects on certain statistical quantities will be now quantified for reactive cases. First, in contrast to inert cases, one can notice that, after the second shock interaction, the vorticity thickness develops less substantially in the presence of bulk viscosity effects, as shown in Fig. 12(a). In addition, it can be noted that the volume 


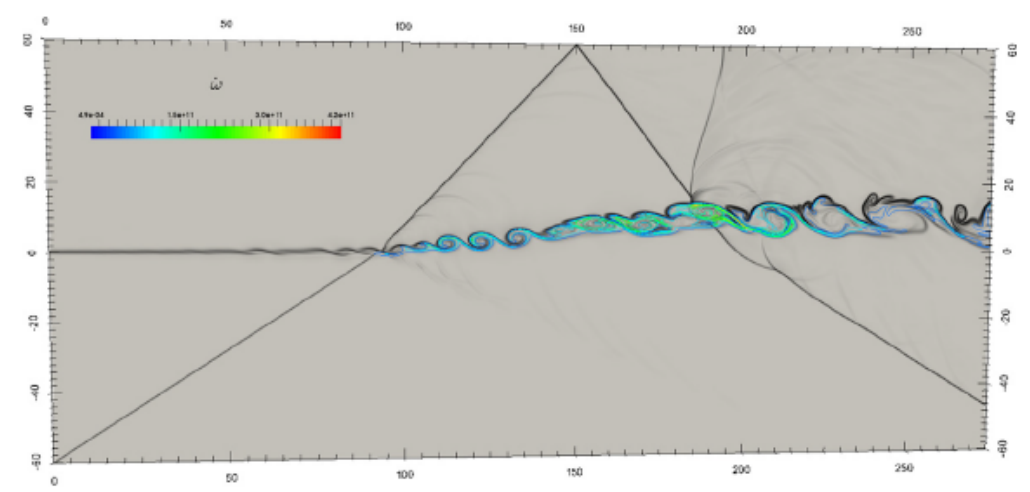

(a) $\kappa \neq 0$

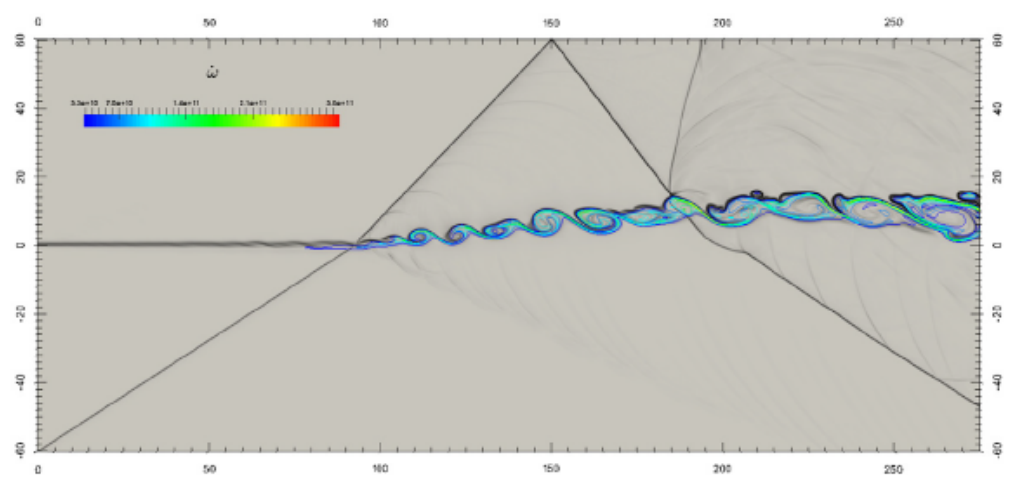

(b) $\kappa=0$

Fig. 11. Heat release rate iso-lines superimposed on a numerical Schlieren image.

viscosity effects seem greater in the region downstream of the reflected shock impact where compressibility effects are the most important.

As far as the evolution of the transverse maxima of the turbulent kinetic energy (TKE) is concerned, the bulk viscosity slightly increases the dissipation of turbulent kinetic energy, especially after the interaction with the second shock in the reactive case, see Fig. 12(b). This observation is consistent with Eq. (2c), an equation that shows the explicit dependency of viscous terms (and resulting dissipation terms) on the ratio $\kappa / \mu$. It could have been expected that the dissipation increases with the bulk viscosity $\kappa$. However, the statistics of the enstrophy - not reported for the sake of conciseness - do display similar trends regardless of bulk viscosity effects.

Reactive as well as non-reactive two-dimensional numerical simulations have shown that the bulk viscosity influences more significantly the instantaneous flowfields than the statistical characteristics of the mixing layer impacted by the oblique shock. In this respect, the longitudinal evolutions of the mean vorticity thickness and turbulent kinetic energy issued from these reactive flow computations only display slight differences compared to inert cases. However, one may expect that these conclusions may be altered by three-dimensional effects and the next section aims at analyzing this possible influence; the question to be answered is as follows: is the three-dimensional configuration more sensitive to the influence of the bulk viscosity than the two-dimensional configuration?

\subsection{Non-reactive three-dimensional mixing layer}

The three-dimensional numerical simulation of the shock wave-mixing layer interaction is illustrated in Fig. 13 which displays iso-values of the numerical Schlieren signal, to delineate the

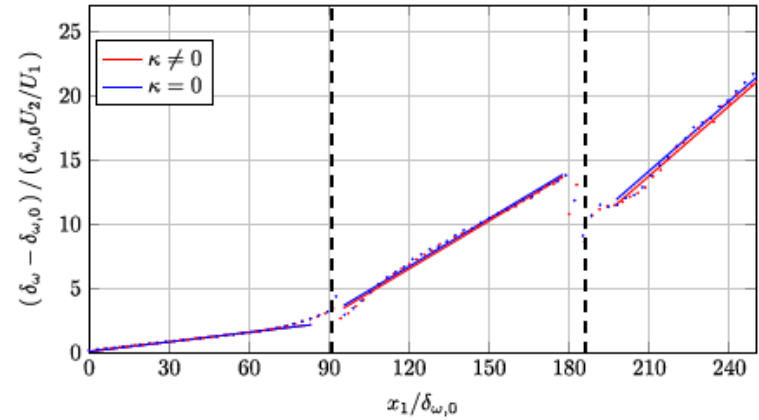

(a)

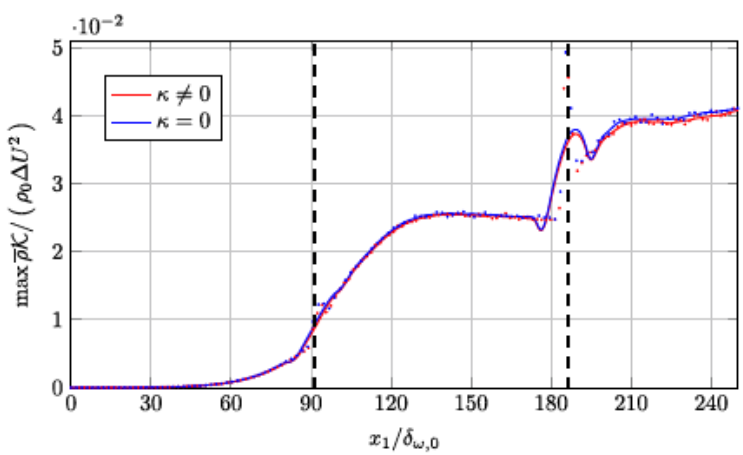

(b)

Fig. 12. Reactive simulation: spatial evolution of the normalized vorticity thickness (a) and normalized turbulent kinetic energy. 


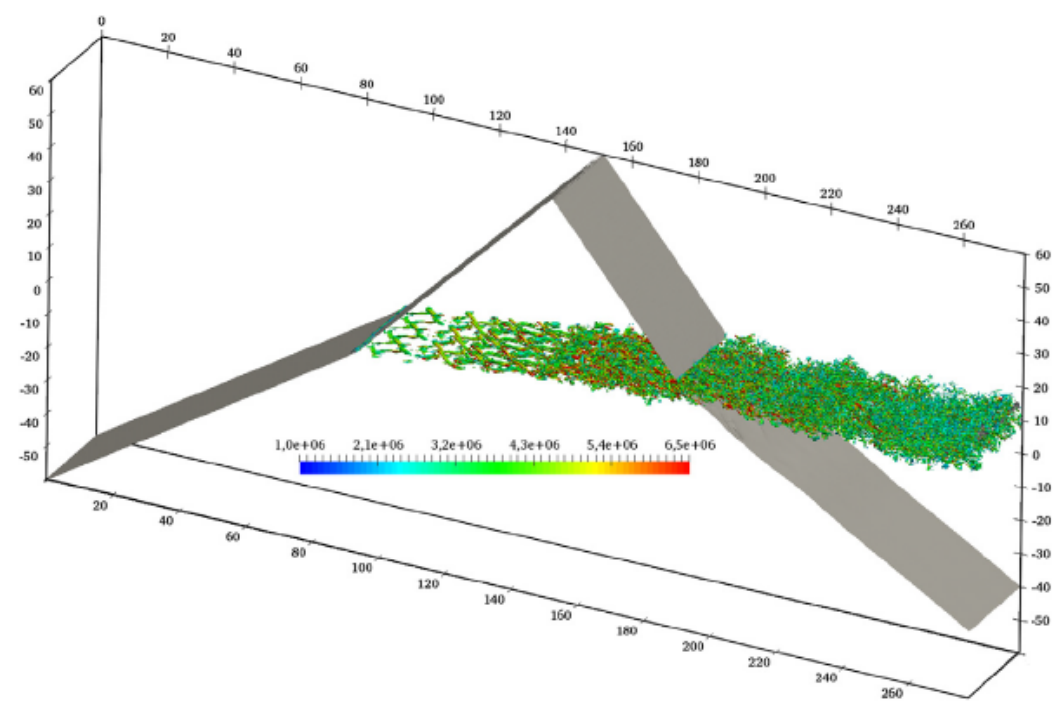

Fig. 13. Three-dimensional case: iso-value of $\lambda_{2}=0.02 \lambda_{2, \max }$ colored by the norm of the vorticity.

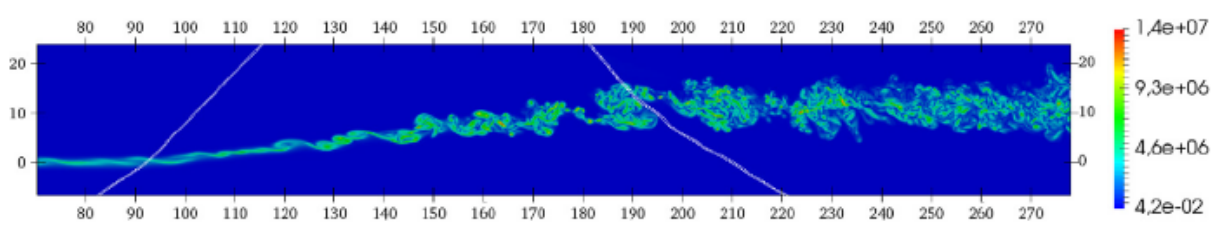

(a) $\kappa \neq 0$

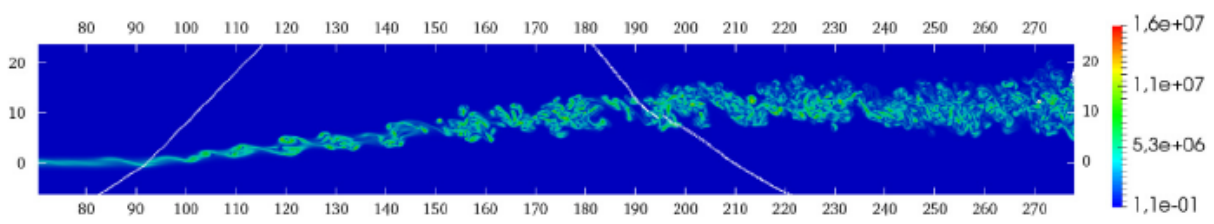

(b) $\kappa=0$

Fig. 14. Iso-lines of the pressure gradient (in white) superimposed on the instantaneous field of vorticity in the $x_{1}-x_{2}$ median plane (i.e., $x_{3}=0$ ).

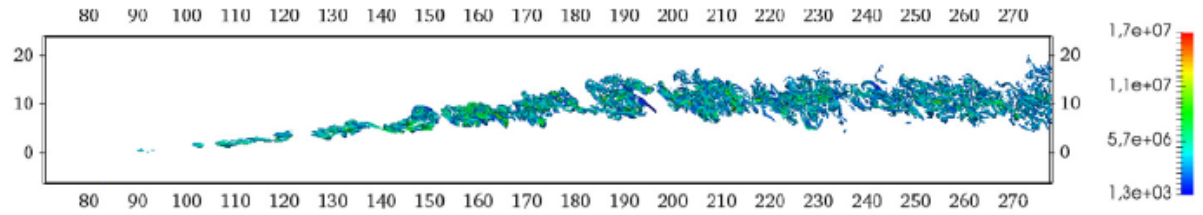

(a) $\kappa \neq 0$

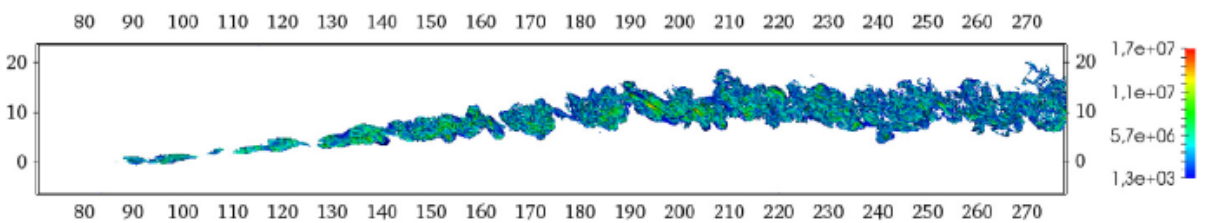

(b) $\kappa=0$

Fig. 15. Instantaneous field of the $Q$-criterion colored by the vorticity in the $x_{1}-x_{2}$ median plane (i.e., $x_{3}=0$ ).

shock waves, together with an iso-value of the $\lambda_{2}$-criterion colored by the norm of the vorticity. Some visualizations are first provided to get preliminary insights into the possible changes that may be induced by the volume viscosity on the structure and topology of the present shock wave-mixing layer interaction. In both cases, i.e., with and without $\kappa$, an intensification of the vortical activity is observed just downstream of the interaction of the mixing layer with the incident oblique shock wave, the mixing layer develops and finally gives rise to a highly three-dimensional dynamics. 


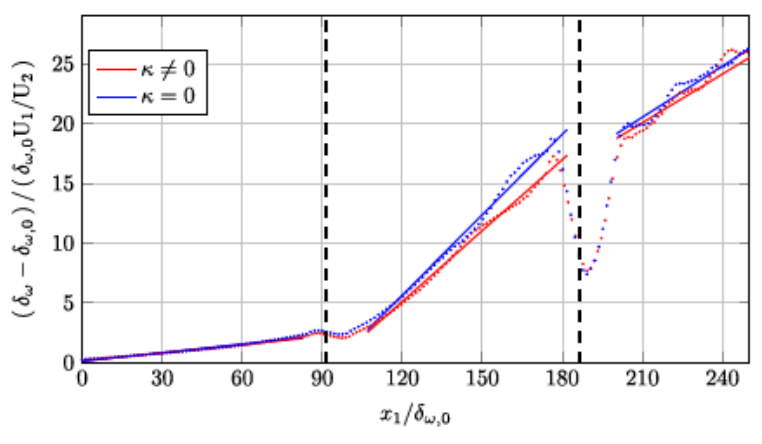

Fig. 16. Evolution of the vorticity thickness in the three-dimensional shock-mixing layer simulation.

Table 3

Values of $\left(\mathrm{U}_{1} / \mathrm{U}_{2}\right) \mathrm{d} \delta_{\omega} / \mathrm{d} x_{1}$ obtained from the three-dimensional mixing layer computations.

\begin{tabular}{llll}
\hline & 1st region & 2nd region & 3rd region \\
\hline$\kappa=0$ & 0.023 & 0.223 & 0.142 \\
$\kappa \neq 0$ & 0.022 & 0.192 & 0.137 \\
\hline
\end{tabular}

Figure 14 displays the instantaneous vorticity field obtained in the median plane $x_{1}-x_{2}$ at normalized time $t \Delta \mathrm{U} / L_{1}=20$. The consideration of the bulk viscosity leads to a substantially different flow topology. Indeed, when $\kappa=0$, there is an earlier formation of vortices taking place just after the interaction with the first shock (i.e., oblique incident shock), as it has been already observed above in the two-dimensional results. However, in the three-dimensional case, the creation of the first vortical structures is much more marked. Figure 15 shows a snapshot of turbulent structures visualized using an iso-value of the $Q$-criterion colored by vorticity. The density of vortical structures is higher in the absence of bulk viscosity. This is especially true after the interaction with the second (i.e., reflected) shock wave where a larger number of localized (and scattered) regions of high vortical activities are visible.

The present three-dimensional numerical simulations have been run over a sufficiently long physical time to generate large databases, which allows for statistical analyses. In addition to this, the extension of the retained computational domains are sufficiently large in the longitudinal direction to reach developed turbulence. The bulk viscosity effect is now analyzed through the vorticity thickness evolution. Figure 16 reports a comparison of this evolution in both cases $\kappa \neq 0$ and $\kappa=0$. Three distinct regions can be delineated, the limits of which are reported in Table 3. Before the first shock, the vorticity thickness growth rate is almost identical in both cases $\kappa=0$ and $\kappa \neq 0$. Between the first and the second shock, it is substantially larger when $\kappa=0$. This inhibition of the mixing layer growth is fully consistent with the instantaneous flow topology discussed above. From the second shock impact location, the mixing layer growth rate changes and takes a value that is smaller than those observed in the second region. In this third region, the vorticity thickness growth rates are similar in both cases.

The profiles of the passive scalar $\xi$ and non-dimensional longitudinal velocity $\left(\tilde{u}_{1}-U_{2}\right) / \Delta U$ are plotted at three different abscissae in Fig. 17 for both cases $\kappa \neq 0$ and $\kappa=0$. The profiles of both quantities remain very close whether the bulk viscosity is taken into account or not. Figure 18 reports the profiles of the mean pressure normalized by its value at the inlet, i.e., $\bar{P} / P_{\text {in }}$, at the same locations. It is noteworthy that the minimum levels achieved by the average pressure are only slightly larger for the case $\kappa \neq 0$. This finding is in line with the previous results of Gonzalez and Emanuel [37] concerning the sensitivity of the pressure field to the

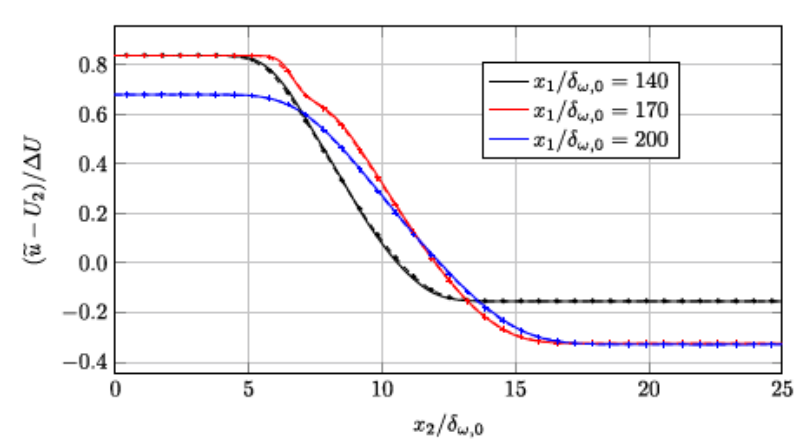

(a)

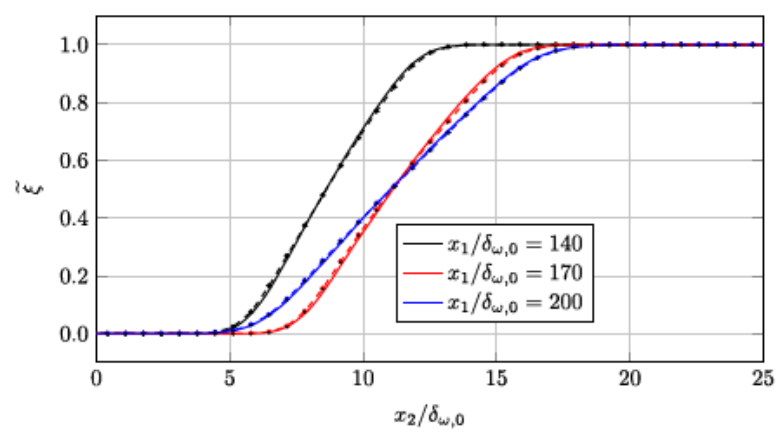

(b)

Fig. 17. Comparison between the normalized velocity and passive scalar profiles obtained for both case $\kappa \neq 0$ and $\kappa=0$ plotted at three abscissae. The solid lines correspond to $\kappa \neq 0$ and the lines with symbol $(++)$ to $\kappa=0$.

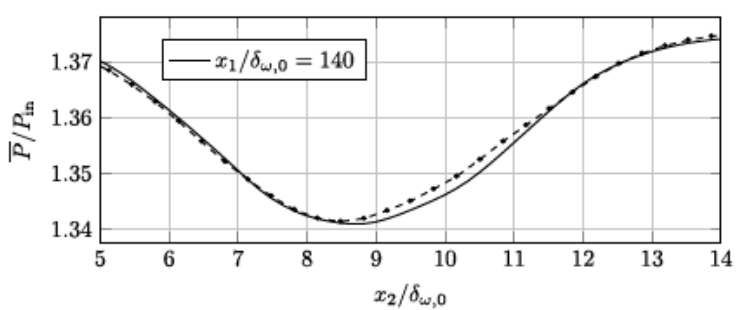

(a)

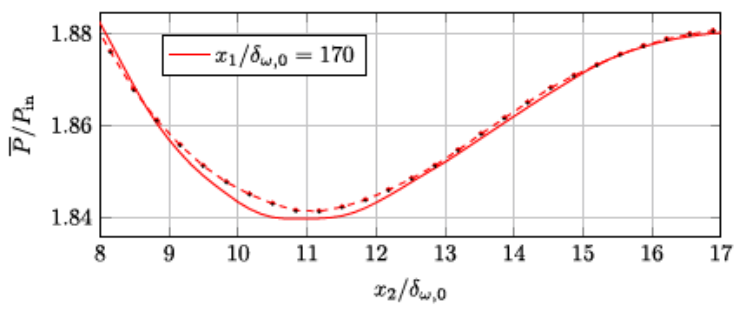

(b)

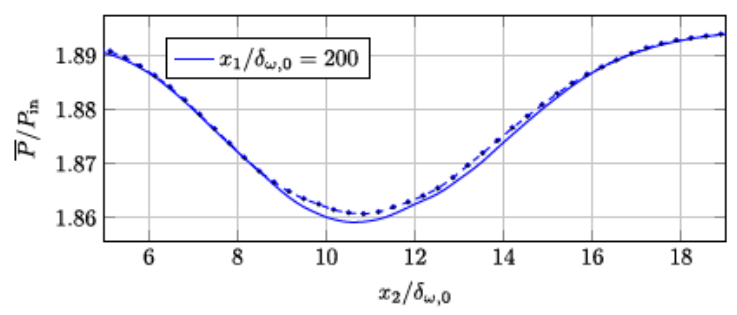

(c)

Fig. 18. Longitudinal profiles of the mean pressure normalized by its value at the inlet $P_{\text {in }}$, same symbols as those used in Fig. 17. 


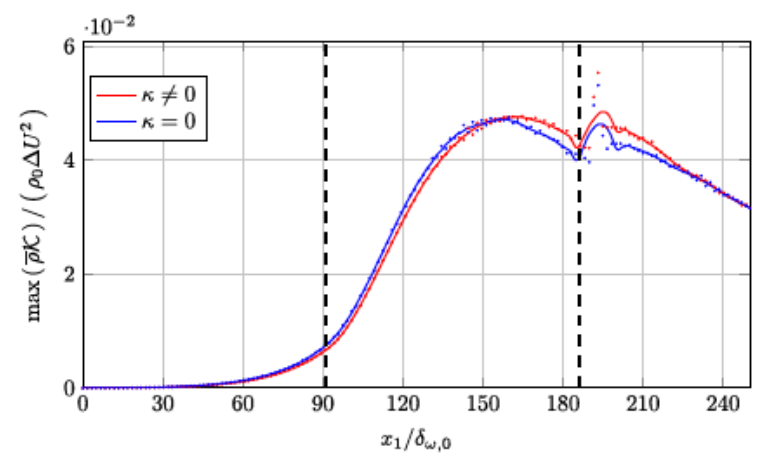

Fig. 19. Spatial evolution of the longitudinal maxima of normalized turbulent kinetic energy.

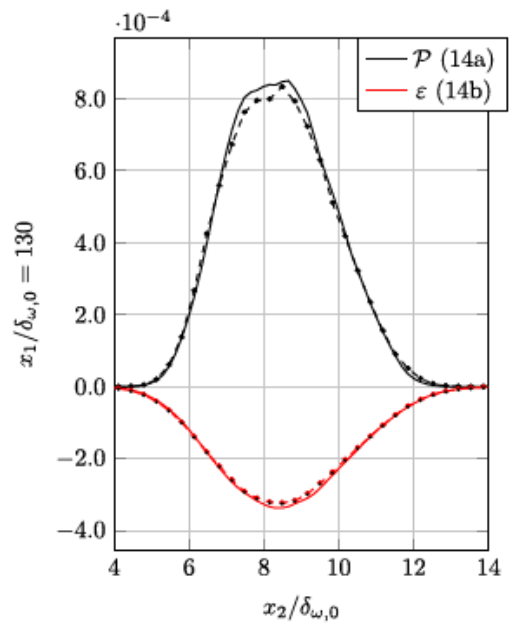

(a)

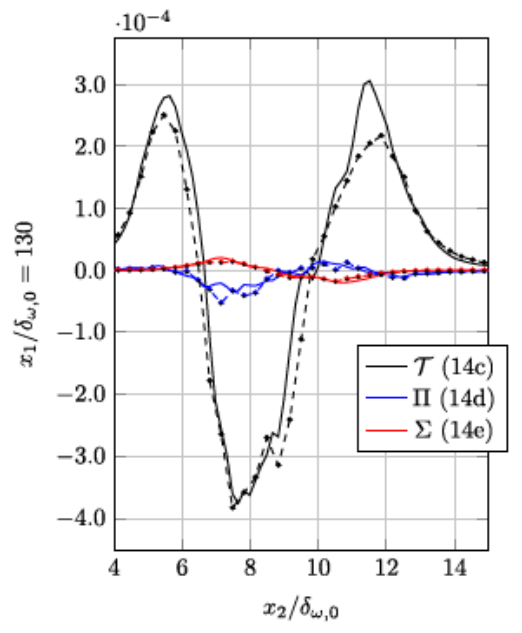

(c)

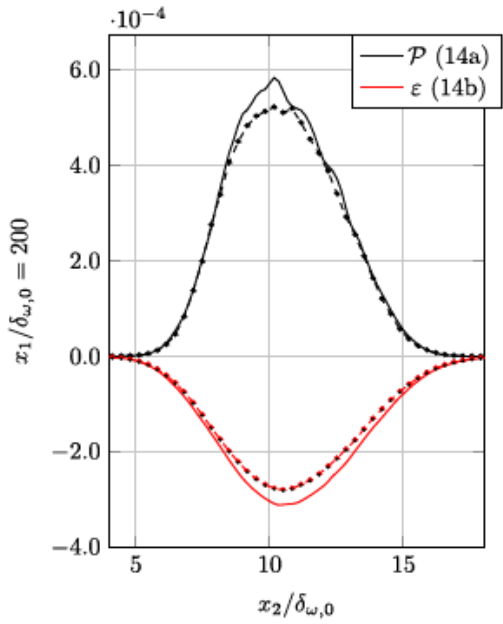

(b)

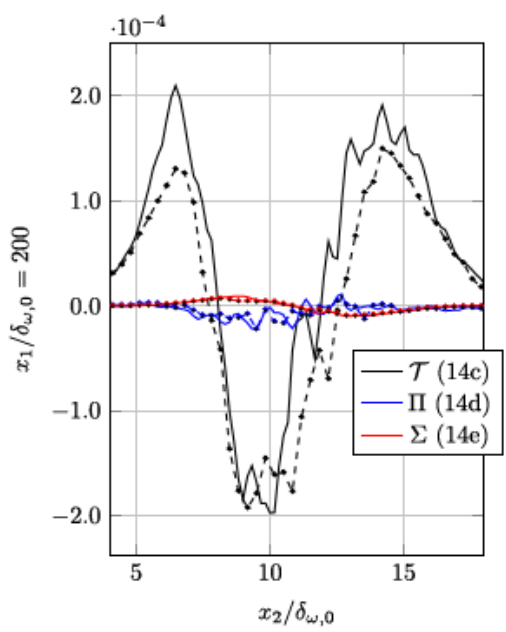

(d)

Fig. 20. TKE budget with all terms normalized by $\Delta \mathrm{U}^{3} / \delta_{\omega, 0}$, same symbols as those retained in Fig. 17.

Stokes hypothesis and the possible modification of the pressure distribution obtained as the ratio $\kappa / \mu$ is increased.

Since it has been shown that the first-order statistical moments do not display a significant sensitivity to the bulk viscosity, a closer look is now taken at some second-order moments that characterize the velocity and passive scalar fluctuations. Figure 19 reports the longitudinal evolution of the maximum values of the normalized TKE. It is noteworthy that the three-dimensional character of the present set of simulations slightly modifies the conclusion that were previously drawn from the two-dimensional computations: the influence of bulk viscosity is noticeable. Up to the abscissa $x_{1} / \delta_{\omega, 0} \approx 150.0$, neglecting the bulk viscosity only leads to a very 


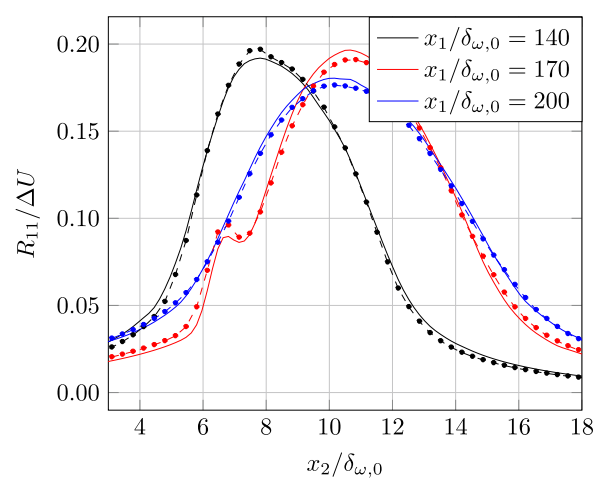

(a)

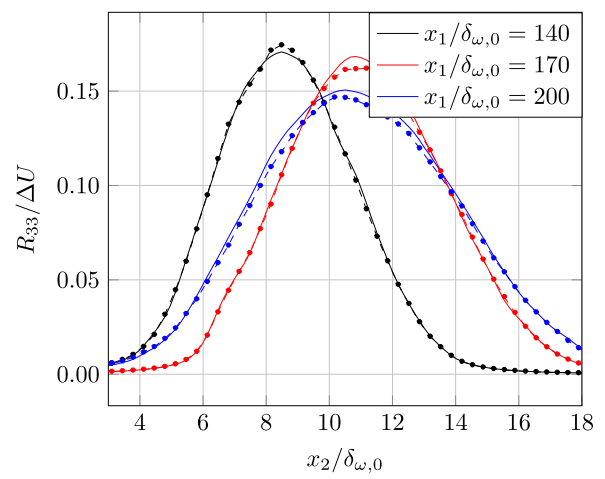

(c)

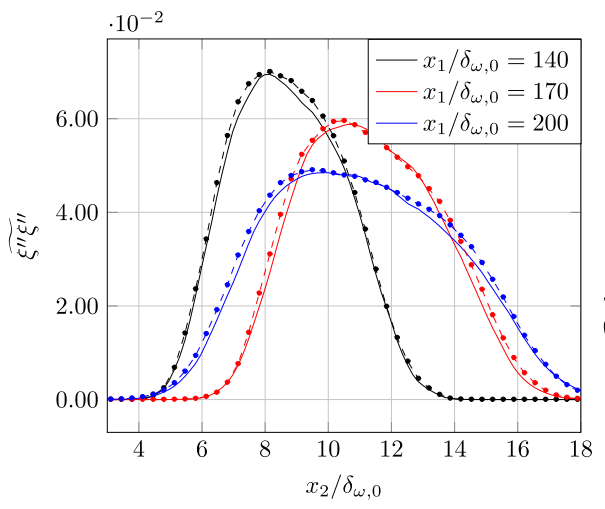

(e)

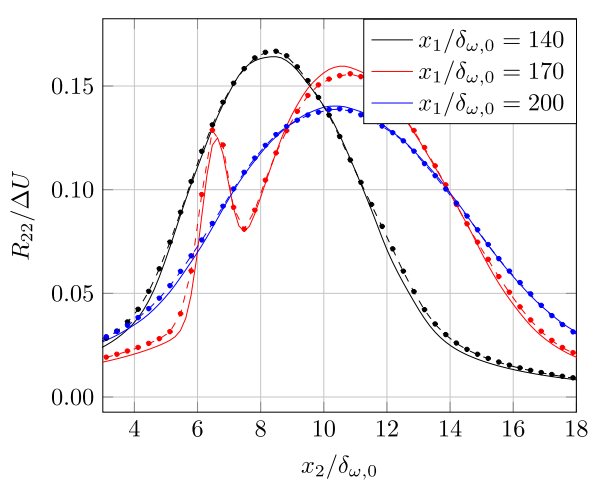

(b)

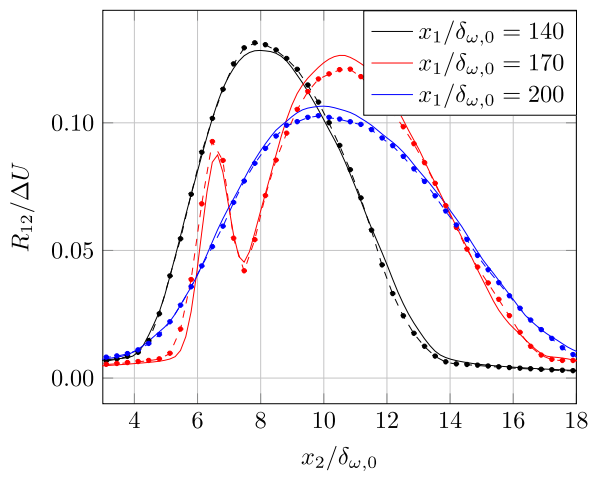

(d)

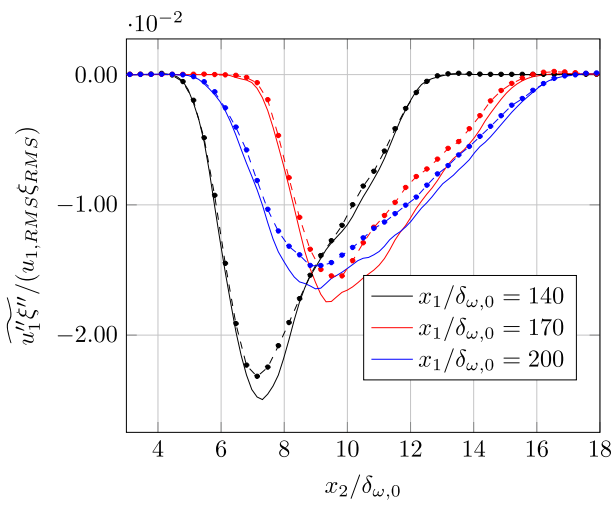

(f)

Fig. 21. Profiles of the Reynolds stress tensor components normalized by $\Delta \mathrm{U}$ together with the mixture fraction variance $\widetilde{\xi^{\prime \prime}} \xi^{\prime \prime}$ and longitudinal component of the scalar flux $\widetilde{u_{1}^{\prime \prime} \xi^{\prime \prime}}$ at three abscissae, same symbols as those retained in Fig. 17.

slight overestimate compared to the case where the effects of $\kappa$ are considered. The region that extends from $x_{1} / \delta_{\omega, 0}=150.0$ until the interaction with the reflected shock is characterized by a significant change of behavior and the values obtained with $\kappa \neq 0$ are larger than those obtained with $\kappa=0$. This region is characterized by strong pressure wave reflection from the upper limit of the computational domain. After the interaction with the reflected shock wave, the maxima of the TKE obtained with or without taking into account the bulk viscosity effects tend to become similar as the end of the computational domain is approached. It can be concluded that, in the absence of the second shock wave interaction and associated parasitic pressure waves issued from the top of the computational domain, the TKE levels would be slightly underestimated if the effects of $\kappa$ were not taken into account. In an attempt to better understand the behavior of the TKE, the analysis of the main terms involved in its transport equation is now carried out.

The transport equation for the turbulent kinetic energy $\mathcal{K}$ is given by

$\partial_{t}(\bar{\rho} \mathcal{K})+\nabla \cdot(\bar{\rho} \widetilde{\boldsymbol{u}} \mathcal{K})=\mathcal{P}+\varepsilon+\mathcal{T}+\Pi+\Sigma$

In this equation, $\mathcal{P}$ is the production term, $\varepsilon$ is the dissipation term, $\mathcal{T}$ denotes the turbulent transport term, $\Pi$ is the pressure-strain term, and finally $\Sigma$ the mass flux term. The budget (12) is deduced from the transport equation of the Reynolds tensor components:

$\frac{\partial\left(\bar{\rho} R_{i j}\right)}{\partial t}+\frac{\partial\left(\bar{\rho} \widetilde{u}_{k} R_{i j}\right)}{\partial x_{k}}=\mathcal{P}_{i j}+\varepsilon_{i j}+\mathcal{T}_{i j}+\Pi_{i j}+\Sigma_{i j}$, 


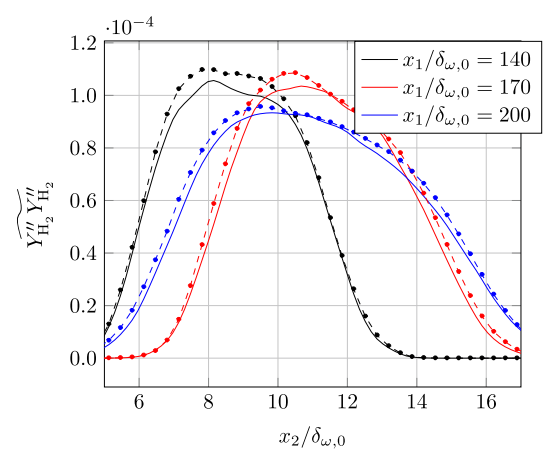

(a)

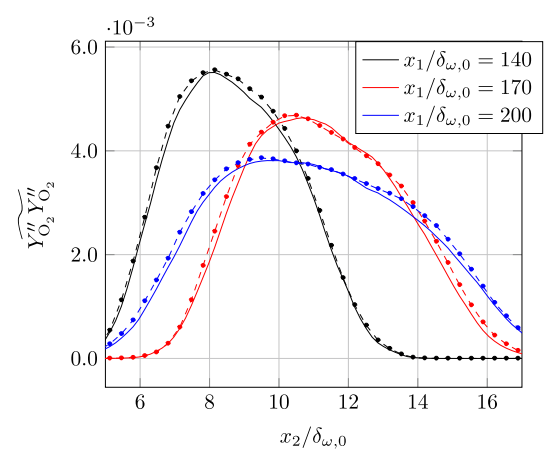

(b)

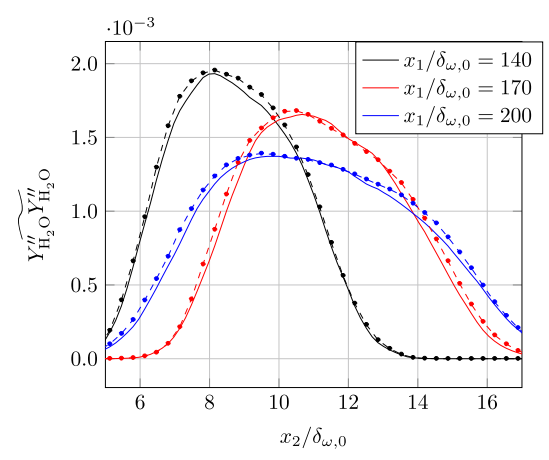

(c)

Fig. 22. Profiles of the variances of chemical species mass fractions at three abscissae, same symbols as those retained in Fig. 17.

with

$$
\left\{\begin{aligned}
\mathcal{P}_{i j}= & -\bar{\rho}\left(R_{i k} \frac{\partial \widetilde{u}_{j}}{\partial x_{k}}+R_{j k} \frac{\partial \widetilde{u}_{i}}{\partial x_{k}}\right), \\
\varepsilon_{i j}= & -\overline{\tau_{i k}^{\prime}} \frac{\partial u_{j}^{\prime \prime}}{\partial x_{k}}-\overline{\tau_{j k}^{\prime} \frac{\partial u_{i}^{\prime \prime}}{\partial x_{k}}}, \\
\mathcal{T}_{i j}= & -\frac{\partial}{\partial x_{k}}\left(\overline{\rho u_{i}^{\prime \prime} u_{j}^{\prime \prime} u_{k}^{\prime \prime}}+\overline{P^{\prime} u_{i}^{\prime \prime}} \delta_{j k}+\overline{P^{\prime} u_{j}^{\prime \prime}} \delta_{i k}-\overline{\tau_{j k}^{\prime} u_{i}^{\prime \prime}}-\overline{\tau_{i k}^{\prime} u_{j}^{\prime \prime}}\right), \\
\Pi_{i j}= & \overline{P^{\prime} \frac{\partial u_{i}^{\prime \prime}}{\partial x_{j}}}+\overline{P^{\prime} \frac{\partial u_{j}^{\prime \prime}}{\partial x_{i}}}, \\
\Sigma_{i j}= & \left(\overline{u_{i}^{\prime \prime}} \frac{\partial \overline{j_{j k}}}{\partial x_{k}}+\overline{u_{j}^{\prime \prime}} \frac{\partial \overline{\tau_{i k}}}{\partial x_{k}}\right)-\left(\overline{u_{i}^{\prime \prime}} \frac{\partial \bar{P}}{\partial x_{j}}+\overline{u_{j}^{\prime \prime}} \frac{\partial \bar{P}}{\partial x_{i}}\right)
\end{aligned}\right.
$$

In the above set of equations, it has been chosen to split the pressure into a mean and a fluctuating part but it should be recognized that there exist other ways to handle pressure terms in the second-order moment transport equations. Here, we are following the same procedure as the one previously considered by Pantano et Sarkar [38]. However, it is acknowledged that, for other conditions related, for instance, to transport modeling in turbulent premixed flames in the flamelet regime, keeping the instantaneous pressure can be a better choice since local flamelet relationships may provide relevant closures for the corresponding effects, see for instance Bray et al. [39] or Robin et al. [40].

The analysis of the main terms involved in the TKE transport equation is carried out at two distinct locations to infer the impact of the volume viscosity. Figure 20 shows that the most important contributions are associated to the production and dissipation terms. Their amplitude is found to be slightly smaller when $\kappa$ is not taken into account. The turbulent transport term is positive at the periphery of the mixing layer while it tends to be negative within the mixing layer. This quantity, which is larger in the case featuring $\kappa \neq 0$, removes energy from regions characterized by large fluctuations levels to transfer it in regions characterized by lower levels of TKE. Figure 20 also shows that the contributions due to pressure-strain and mass flux terms remain negligible compared to the others, for both cases. 
Figure 21 reports the distribution of the Reynolds stress components together with the variance of the passive scalar and the scalar flux components for both cases $\kappa \neq 0$ and $\kappa=0$. The three streamwise positions under consideration are representative of the variations observed on the TKE profile reported in Fig. 19. The profiles of the Reynolds stress components show that the maxima of its diagonal components follow the trends reported in Fig. 19. Figure 21(f), which displays the longitudinal evolution of the scalar flux component $\widetilde{u_{1}^{\prime \prime} \xi^{\prime \prime}} /\left(u_{1, \text { RMS }} \xi_{\text {RMS }}\right)$, reveals that the maximum value of the correlation between the longitudinal velocity fluctuation and the scalar fluctuation is slightly underestimated when the effects of bulk viscosity are not considered.

Figure 22 reports the variance of the mass fractions of chemical species present in the mixture. The hydrogen, which is characterized by the highest ratio $\kappa / \mu$ is the one that displays the largest differences (up to approximately ten percent) between the two cases, i.e., $\kappa=0$ and $\kappa \neq 0$. The differences observed at the three locations concern both the shape and maximum levels, which depend on the species under consideration. Indeed, it is found that the distribution of the profiles for all chemical species is slightly wider - indicating that the fluid is incorporated more markedly when the effects of bulk viscosity are not taken into account, which leads to a reduction of fluctuations around the averaged value. A similar effect is observed when the convective Mach number values are increased [26,41].

\section{Summary and conclusions}

In the present manuscript, two- and three-dimensional numerical simulations of spatially-developing compressible mixing layers impacted by an oblique shock wave are conducted for a convective Mach number $M_{c}=0.48$. The emphasis is placed on the possible influence of the bulk viscosity on the mixing processes. Thus, a mixture of hydrogen and air is considered in conditions that are representative of experimental benchmarks relevant to high-speed flow combustion. In a first step of the analysis, twodimensional computations of inert and reactive mixing layers are performed. A significant impact of the bulk viscosity is observed on the instantaneous flowfields while averaged quantities do not exhibit any remarkable modification. It is also worth noting that the reactive cases only display slight differences with respect to inert cases: this is especially true for the longitudinal evolutions of the vorticity thickness and turbulent kinetic energy. Threedimensional simulations of inert mixing layers are subsequently conducted. The influence of the bulk viscosity is more visible in these three-dimensional cases: it tends to reduce the mixing layer growth rate compared to the case where it is not taken into account. The comparison is also performed in terms of higherorder statistical moments. This last part of the analysis shows that the bulk viscosity effects tend to amplify the velocity gradients at the boundaries of the mixing layer, and consequently favor the return to equilibrium. From the above synthesis of the obtained results, one may expect that refined large-eddy simulations (LES) may be rather sensitive to the consideration of bulk viscosity, while Reynolds-averaged Navier-Stokes (RANS) simulations, which are based on statistical averages, are not. One possible perspective of the present work concerns the filtering of the present dataset, which may provide further insights so as to assess this conclusion. Finally, from the present set of results, it is recommended to take the bulk viscosity effects into account especially when highlyresolved large-eddy simulations (LES) are considered.

\section{Acknowledgments}

The computations were performed using the High Performance Computing resources from the MÉSOCENTRE DE CALCUL POITEVIN and from GENCI under allocations x20142a0912 and x20142b7251. The first author benefited from interesting discussions with Aimad Erraiy. This work has been presented at the tenth International Conference on Computational Fluid Dynamics (Barcelona, July 2018).

\section{References}

[1] U. Balucani, M. Zoppi, Dynamics of the Liquid State, Vol. 10, Clarendon Press 1995.

[2] M.S. Cramer, Numerical estimates for the bulk viscosity of ideal gases, Phys Fluids 24 (2012) 066102.

[3] G. Billet, V. Giovangigli, G. De Gassowski, Impact of volume viscosity on a shock-hydrogen-bubble interaction, Combust. Theory Model. 12 (2) (2008) 221-248.

[4] S. Chapman, T. Cowling, The Mathematical Theory of Non-uniform Gases, Cambridge University Press, 1970

[5] A. Ern, V. Giovangigli, Volume viscosity of dilute polyatomic gas mixtures, European J. Mech. B 14 (5) (1995) 653-669.

[6] A. Ern, V. Giovangigli, Fast and accurate multicomponent transport property evaluation, J. Comput. Phys. 120 (1995) 105-116.

[7] A. Ern, V. Giovangigli, EGlib users manual, 1996.

[8] G. Fru, G. Janiga, D. Thévenin, Impact of volume viscosity on the structure of turbulent premixed flames in the thin reaction zone regime, Flow Turbul Combust. 88 (4) (2012) 451-478.

[9] M.S. Cramer, F. Bahmani, Effect of large bulk viscosity on large-Reynoldsnumber flows, J. Fluid Mech. 751 (2014) 142-163.

[10] F. Bahmani, M.S. Cramer, Suppression of shock-induced separation in fluids having large bulk viscosities, J. Fluid Mech. 756 (2014) 142-163.

[11] A.V.Chikitkin, B.V. Rogov, G.A. Tirsky, S.V.Utyuzhnikov, Effect of bulk viscosity in supersonic flow past spacecraft, Appl. Numer. Math. 93 (2015) 47-60.

[12] C. Huete, A.L. Sánchez, F.A. Williams, Diffusion-flame ignition by shock-wave impingement on a hydrogen/air supersonic mixing layer, J. Propul. Power 33 (2017) 256-263.

[13] R. Buttay, G. Lehnasch, A. Mura, Analysis of small-scale scalar mixing processes in highly under-expanded jets, Shock Waves 26 (2) (2016) 93-212.

[14] R. Boukharfane, Z. Bouali, A. Mura, Evolution of scalar and velocity dynamics in planar shock-turbulence interaction, Shock Waves 28 (2018) 1117-1141.

[15] P.J. Martínez Ferrer, R. Buttay, G. Lehnasch, A. Mura, A detailed verification procedure for compressible reactive multicomponent Navier-Stokes solvers, Comput. \& Fluids 89 (2014) 88-110.

[16] J. Lin, C. Scalo, L. Hesselink, High-fidelity simulation of a standing-wave thermoacoustic-piezoelectric engine, J. Fluid Mech. 808 (2016) 19-60.

[17] G.J. Prangsma, A.H. Alberga, J.J.M. Beenakker, Ultrasonic determination of the volume viscosity of $\mathrm{N}_{2}, \mathrm{CO}, \mathrm{CH}_{4}$, and $\mathrm{CD}_{2}$ between 77 and $300 \mathrm{~K}$, Physica 64 (2) (1973) 278-288

[18] D. Bruno, V. Giovangigli, Relaxation of internal temperature and volume viscosity, Phys. Fluids 23 (2011) 093104

[19] A. Ern, V. Giovangigli, Multicomponent Transport Algorithms, Vol. 24 Springer Science \& Business Media, 1994.

[20] L. Monchick, E.A. Mason, Transport properties of polar gases, J. Chem. Phys 35 (5) (1961) 1676-1697.

[21] A. Ern, V. Giovangigli, EGlib: A general-purpose fortran library for multicomponent transport property evaluation. Technical report, Manual EGlib Version, 2004.

[22] A.C. Hindmarsh, P.N. Brown, K.E. Grant, S.L. Lee, R. Serban, D.E. Shumaker, C.S Woodward, Sundials: Suite of nonlinear and differential/algebraic equation solvers, ACM Trans. Math. Software 31 (3) (2005) 363-396.

[23] J.L. Ziegler, R. Deiterding, J.E. Shepherd, D.I. Pullin, An adaptive high-order hybrid scheme for compressible, viscous flows with detailed chemistry, J. Comput. Phys. 230 (20) (2011) 7598-7630.

[24] R. Boukharfane, F.H.E. Ribeiro, Z. Bouali, A. Mura, A combined ghost-pointforcing/direct-forcing immersed boundary method (IBM) for compressible flow simulations, Comput. \& Fluids 162 (2018) 91-112.

[25] J.D. Ramshaw, Simple model for mixing at accelerated fluid interfaces with shear and compression, Phys. Rev. E 61 (5) (2000) 5339.

[26] P.J. Martínez Ferrer, G. Lehnasch, A. Mura, Compressibility and heat release effects in high-speed reactive mixing layers. Growth rates and turbulence characteristics, Combust. Flame 180 (2017) 284-303.

[27] C. Pantano, S. Sarkar, F.A. Williams, Mixing of a conserved scalar in a turbulent reacting shear layer, J. Fluid Mech. 481 (2003) 291-328.

[28] R.E. Mitchell, R.J. Kee, General-purpose computer code for predicting chemical-kinetic behavior behind incident and reflected shocks. Technical report, Sandia National Labs., Livermore, CA (USA), 1982.

[29] M. Ó Conaire, H.J. Curran, J.M. Simmie, W.J. Pitz, C.K. Westbrook, A comprehensive modeling study of hydrogen oxidation, Int. J. Chem. Kinet. 36 (11) (2004) 603-622.

[30] R. Buttay, G. Lehnasch, A. Mura, Turbulent mixing and molecular transport in highly under-expanded hydrogen jets, Int. J. Hydrogen Energy 43 (17) (2018) 8488-8505. 
[31] R. Buttay, L. Gomet, G. Lehnasch, A. Mura, Highly resolved numerical simulation of combustion downstream of a rocket engine igniter, Shock Waves 27 (4) (2017) 655-674.

[32] S.B. Pope, Turbulent Flows, Cambridge University Press, 2000.

[33] M. Brouillette, The Richtmyer-Meshkov instability, Annu. Rev. Fluid Mech. 34 (1) (2002) 445-468.

[34] Y. Yan, C. Chen, P. Lu, C. Liu, Study on shock wave-vortex ring interaction by the micro vortex generator controlled ramp flow with turbulent inflow, Aerosp. Sci. Technol. 30 (1) (2013) 226-231.

[35] C.D. Pierce, Progress-variable Approach for Large-eddy Simulation of Turbulent Combustion (Ph.D. thesis), Stanford University, 2001.

[36] L. Gomet, V. Robin, A. Mura, A multiple-inlet mixture fraction model for non premixed combustion, Combust. Flame 162 (2015) 668-687.
[37] H. Gonzalez, G. Emanuel, Effect of bulk viscosity on Couette flow, Phys. Fluids A 5 (5) (1993) 1267-1268.

[38] C. Pantano, S. Sarkar, A study of compressibility effects in the high-speed turbulent shear layer using direct simulation, J. Fluid Mech. 451 (2002) 329371.

[39] K. Bray, M. Champion, P. Libby, Premixed flames in stagnating turbulence. Part IV: a new theory for Reynolds stresses and Reynolds fluxes applied to impinging flows, Combust. Flame 120 (2000) 1-18.

[40] V. Robin, M. Champion, A. Mura, A second-order model for turbulent reactive flows with variable equivalence ratio, Combust. Sci. Technol. 180 (2008) 1707-1732.

[41] I. Mahle, Direct and Large-eddy Simulation of Inert and Reacting Compressible Turbulent Shear Layers (Ph.D. thesis), Technische Universität München, 2007. 